\title{
离 PENTAGONS
}

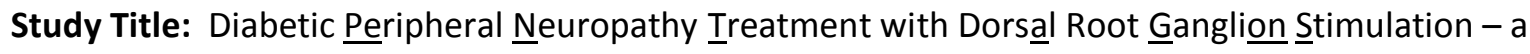
Randomised Controlled Trial.

Internal Reference Number / Short title: PENTAGONS

Ethics Ref: 18/SC/0146

Date and Version No: V4.0_31Jul2019

\section{Chief Investigator:}

Investigators:

\section{Professor James FitzGerald}

Nuffield Department of Surgical Sciences, University of Oxford james.fitzgerald@nds.ox.ac.uk

07967645273

PA Vicky Ford tel. 01865234605

Prof Sam Eldabe, Professor of Pain Medicine, South Tees Hospitals NHSFT

Mr Girish Vajramani, Department of Neurosurgery, University Hospital Southampton NHSFT

Mr Alexander Green, Nuffield Department of Surgical Sciences, University of Oxford

Dr Abdul Lalkhen, Salford Royal NHS Foundation Trust

Mr Erlick Pereira, Consultant Neurosurgeon, St George's University Hospital, London

Miss Stana Bojanic, Department of Neurosurgery, Oxford University Hospitals NHSFT

Dr Rustam Rea, Oxford Centre for Diabetes, Endocrinology and Metabolism

PENTAGONS: Diabetic Peripheral Neuropathy Treatment with Dorsal Root Ganglion Stimulation.

IRAS Project ID: 226499 REC Reference: 18/SC/0146

PENTAGONStrial_Protocol_V4.0_31Jul2019.docx31Jul2019

Clinical Research Protocol Template version 13.0

(C) Copyright: The University of Oxford and Oxford University Hospitals NHS Foundation Trust 2016 
$$
\text { 偣 PENTAGONS }
$$

Dr Jane Wolstenholme, Nuffield Department of Population Health, University of Oxford

Susan Dutton, Oxford Clinical Trials Research Unit, University of Oxford

Dr Simon Yarrow, Nuffield Department of Anaesthetics, University of Oxford

Dr Simon Prangnell, Russell Cairns Unit, Oxford University Hospitals NHSFT

Mr Rodrigo Ortega-Garcia, Department of Neurosurgery, Oxford University Hospitals NHSFT

Ms Tamara Edwards, Department of Neurosurgery, Oxford University Hospitals NHSFT

Sponsor: $\quad$ University of Oxford

Funder: $\quad$ Abbott Laboratories (formally known as St Jude Medical, Neurosciences Division). See Appendix C.
\end{abstract}

Chief Investigator Signature:

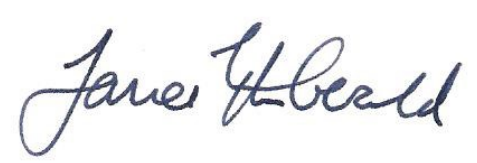

\title{
Conflict of Interest Declaration:
}

The $\mathrm{Cl}$ has received consultancy fees from St Jude Medical, which has now been acquired by Abbott (who are funding this study)

\section{Confidentiality Statement}

This document contains confidential information that must not be disclosed to anyone other than the Sponsor, the Investigator Team, host organisation, and members of the Research Ethics Committee, unless authorised to do so.

PENTAGONS: Diabetic Peripheral Neuropathy Treatment with Dorsal Root Ganglion Stimulation.

IRAS Project ID: 226499 REC Reference: 18/SC/0146

PENTAGONStrial_Protocol_V4.0_31Jul2019.docx31Jul2019

Clinical Research Protocol Template version 13.0

(C) Copyright: The University of Oxford and Oxford University Hospitals NHS Foundation Trust 2016

Page 2 of 46 


\section{Ao PENTAGONS}

\section{TABLE OF CONTENTS}

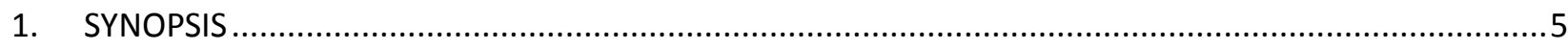

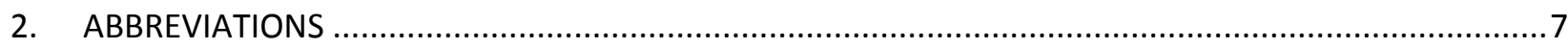

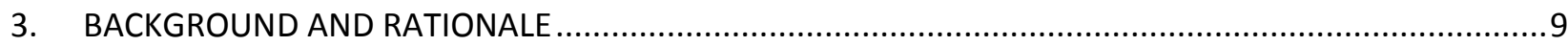

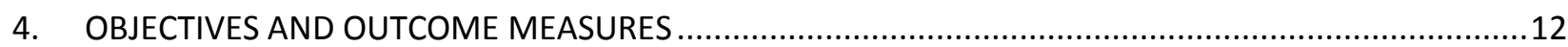

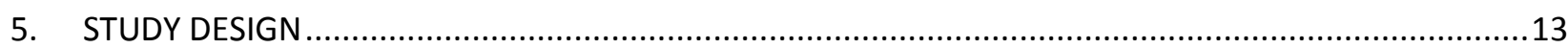

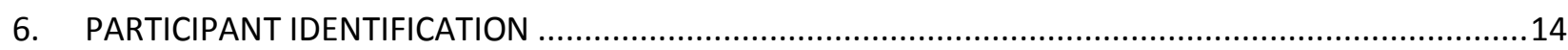

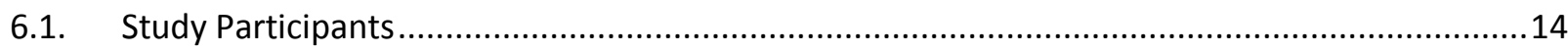

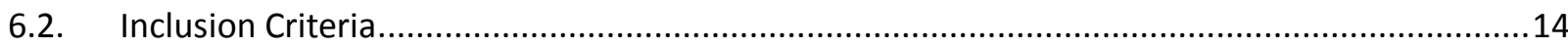

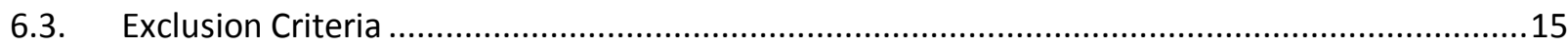

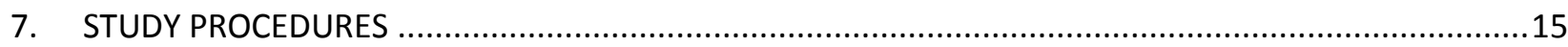

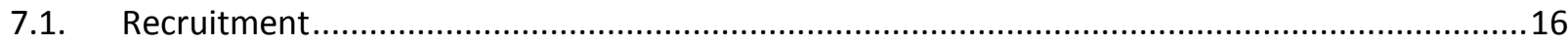

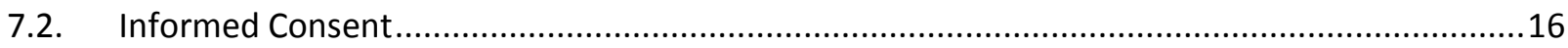

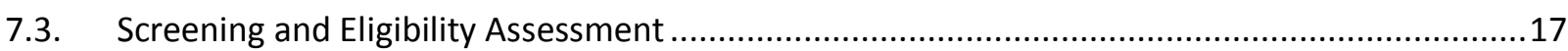

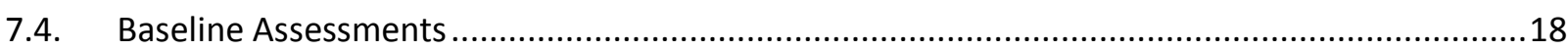

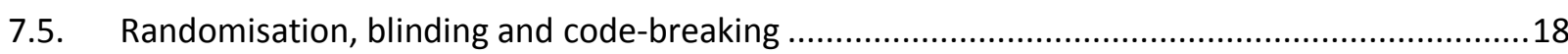

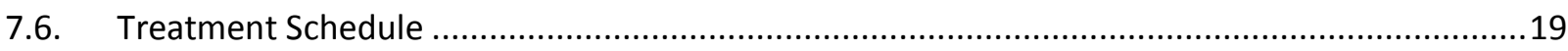

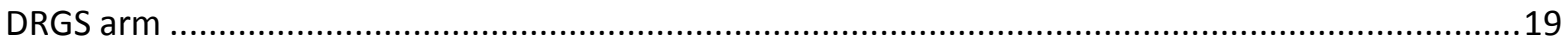

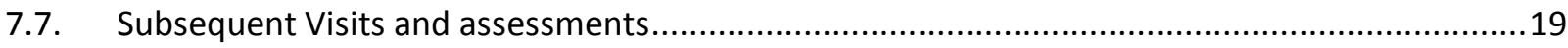

DRGS Arm: Participants allocated DRGS will be followed up at the following time points: ..............19

CMM Arm: Participants allocated CMM will be followed up at the following time points:...............20

7.8. Withdrawal from the Randomised Intervention and Withdrawal from Follow-Up ...................21

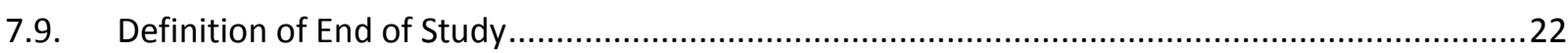

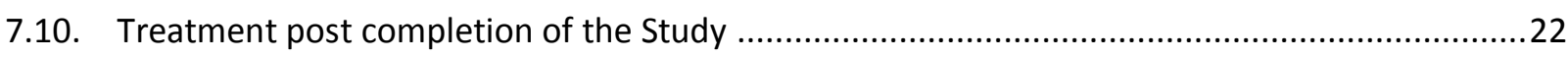

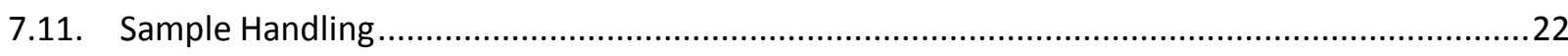

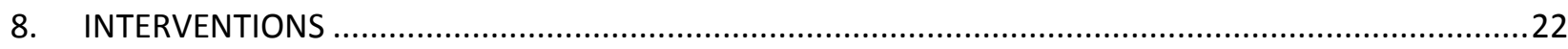

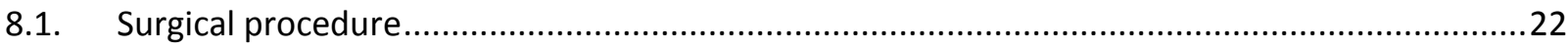

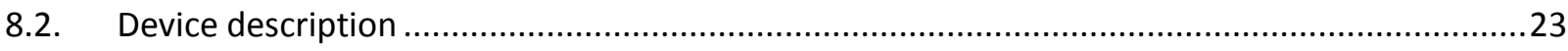

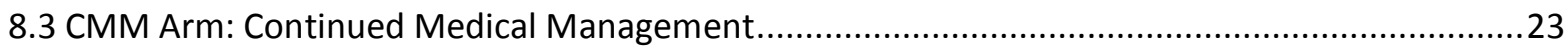

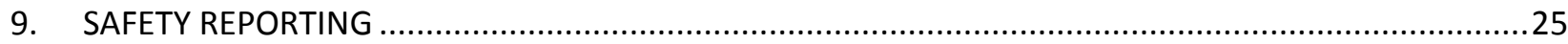

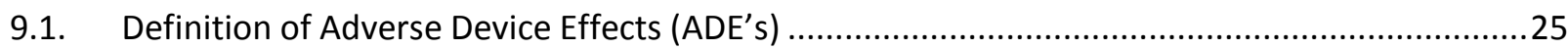

PENTAGONS: Diabetic Peripheral Neuropathy Treatment with Dorsal Root Ganglion Stimulation.

IRAS Project ID: 226499 REC Reference: 18/SC/0146

PENTAGONStrial_Protocol_V4.0_31Jul2019.docx31Jul2019

Clinical Research Protocol Template version 13.0

(C) Copyright: The University of Oxford and Oxford University Hospitals NHS Foundation Trust 2016 


\section{全 PENTAGONS}

The following list of ADE's, in relation to the DRGS procedure, will be reported:

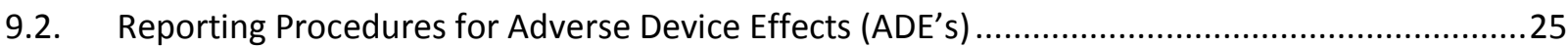

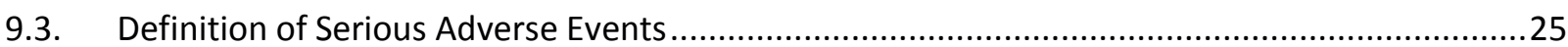

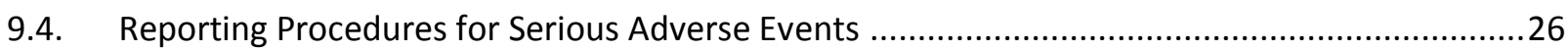

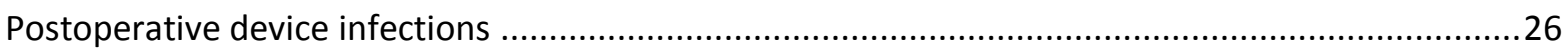

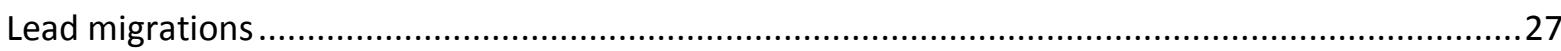

9.5. Reporting Procedures for Unexpected Serious Adverse Events (SUSAR) and Unanticipated

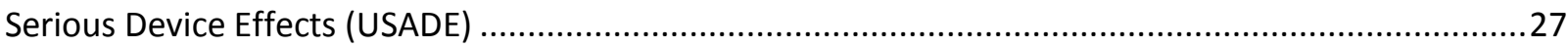

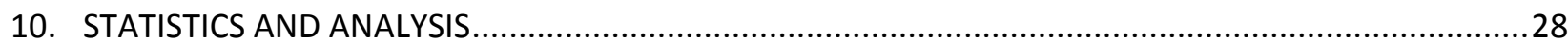

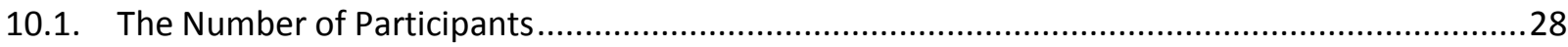

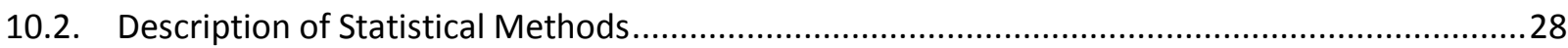

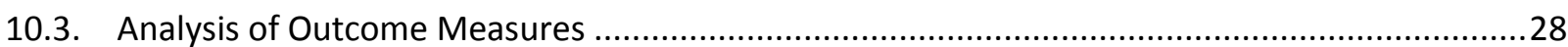

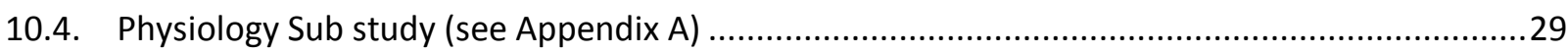

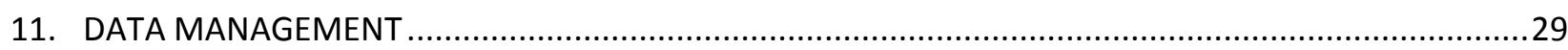

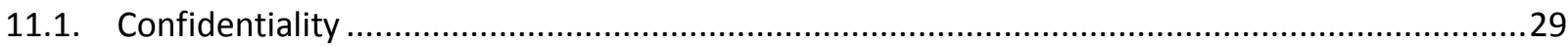

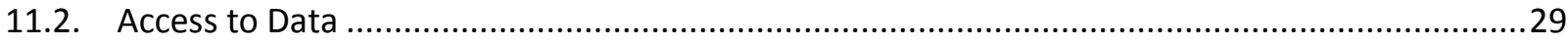

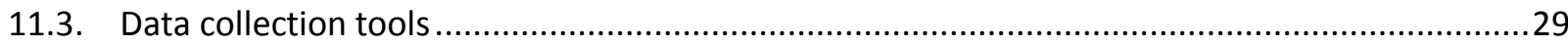

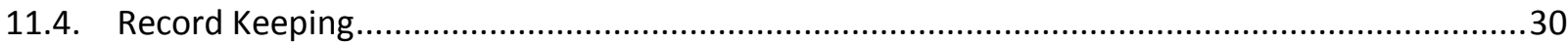

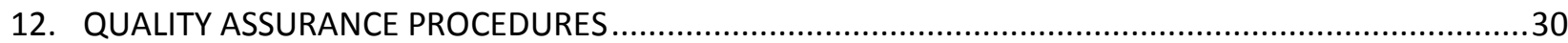

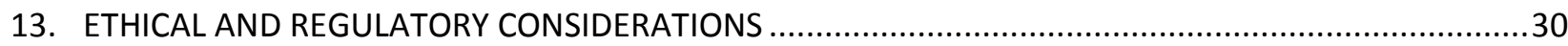

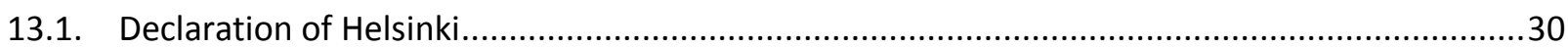

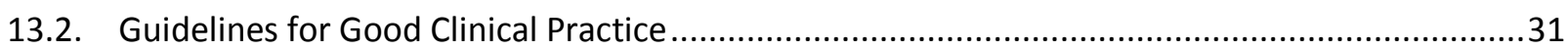

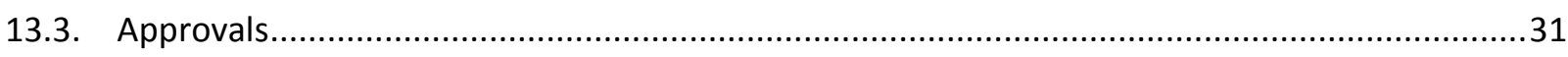

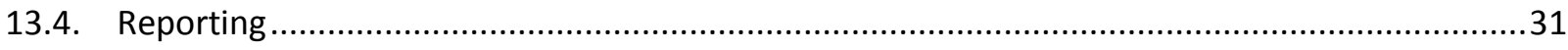

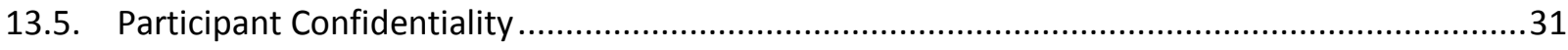

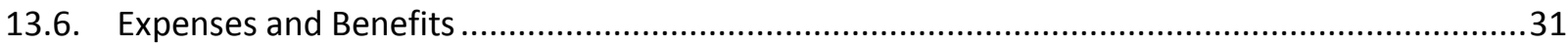

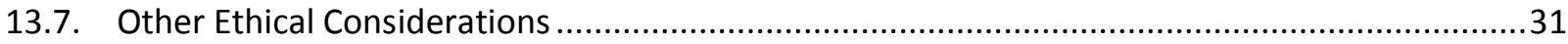

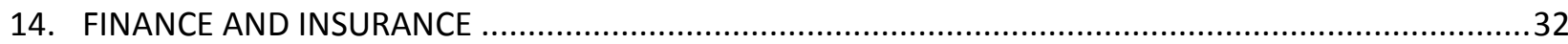

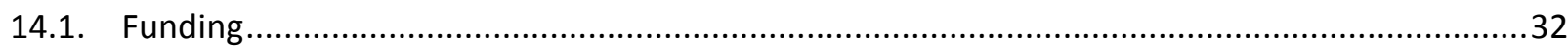

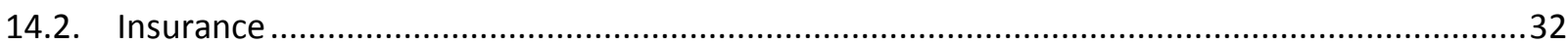

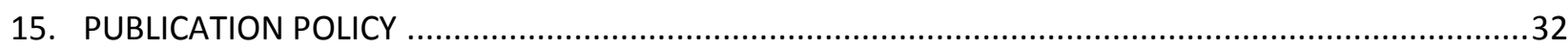

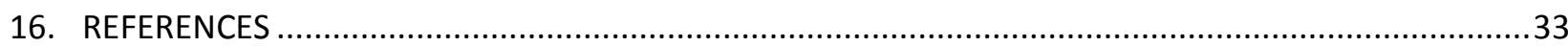

PENTAGONS: Diabetic Peripheral Neuropathy Treatment with Dorsal Root Ganglion Stimulation.

IRAS Project ID: 226499 REC Reference: 18/SC/0146

PENTAGONStrial_Protocol_V4.0_31Jul2019.docx31Jul2019

Clinical Research Protocol Template version 13.0

(C) Copyright: The University of Oxford and Oxford University Hospitals NHS Foundation Trust 2016 


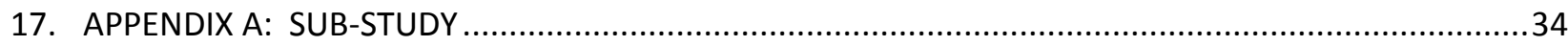

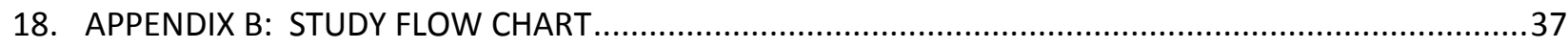

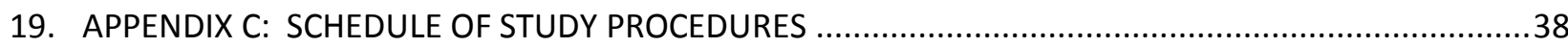

20. APPENDIX D: MICHIGAN NEUROPATHY SCREENING INSTRUMENT (MNSI) .......................................40

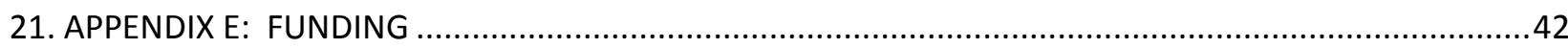

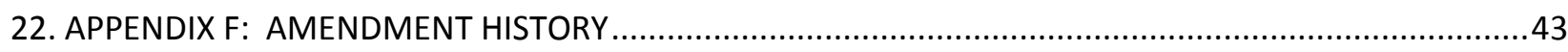

1. SYNOPSIS

\begin{tabular}{|c|c|c|}
\hline Study Title & \multicolumn{2}{|c|}{$\begin{array}{l}\text { Diabetic PEripheral Neuropathy Treatment with dors } \underline{\text { Al Root }} \text { GangliON } \\
\text { Stimulation - a Randomised Controlled Trial. }\end{array}$} \\
\hline $\begin{array}{l}\text { Internal ref. no. / short } \\
\text { title }\end{array}$ & \multicolumn{2}{|l|}{ PENTAGONS } \\
\hline Study Design & \multicolumn{2}{|c|}{$\begin{array}{l}\text { Randomised open label, multi-centre, two-arm trial of Dorsal Root } \\
\text { Ganglion Stimulation (DRGS) versus continued medical management } \\
\text { (CMM) }\end{array}$} \\
\hline Study Participants & \multicolumn{2}{|c|}{$\begin{array}{l}\text { Patients with medication-resistant severe pain due to diabetic peripheral } \\
\text { neuropathy affecting the feet. }\end{array}$} \\
\hline Planned Sample Size & \multicolumn{2}{|c|}{$\begin{array}{l}56 \text { randomised ( } 28 \text { in surgery arm and } 28 \text { in continued medical } \\
\text { management arm (CMM)) } \\
20-24 \text { of the patients in the main trial ( } 10-12 \text { DRGS and } 10-12 \mathrm{CMM} \text { ) at the } \\
\text { lead centre will additionally be recruited into a sub study examining the } \\
\text { physiological effects of DRGS in this patient population. }\end{array}$} \\
\hline \multirow[t]{2}{*}{ Planned Study Period } & \multicolumn{2}{|l|}{2 years } \\
\hline & Objectives & Outcome Measures \\
\hline Primary & $\begin{array}{l}\text { To establish the efficacy of DRGS for } \\
\text { pain relief in Painful Diabetic } \\
\text { Neuropathy of the feet. }\end{array}$ & $\begin{array}{l}\text { Percentage of patients obtaining } \\
\text { at least } 50 \% \text { reduction in pain } \\
\text { visual analogue scale (VAS) score } \\
\text { at } 30 \text { weeks post randomisation, } \\
\text { compared to baseline pre- } \\
\text { randomisation values }\end{array}$ \\
\hline Secondary & As above & $\begin{array}{l}\text { Pain VAS scores at } 8 \text { and } 18 \\
\text { weeks } \\
\text { Brief Pain Inventory (BPI) and } \\
\text { Neuropathic Pain Symptom }\end{array}$ \\
\hline
\end{tabular}

PENTAGONS: Diabetic Peripheral Neuropathy Treatment with Dorsal Root Ganglion Stimulation.

IRAS Project ID: 226499 REC Reference: 18/SC/0146

PENTAGONStrial_Protocol_V4.0_31Jul2019.docx31Jul2019

Clinical Research Protocol Template version 13.0

(C) Copyright: The University of Oxford and Oxford University Hospitals NHS Foundation Trust 2016 


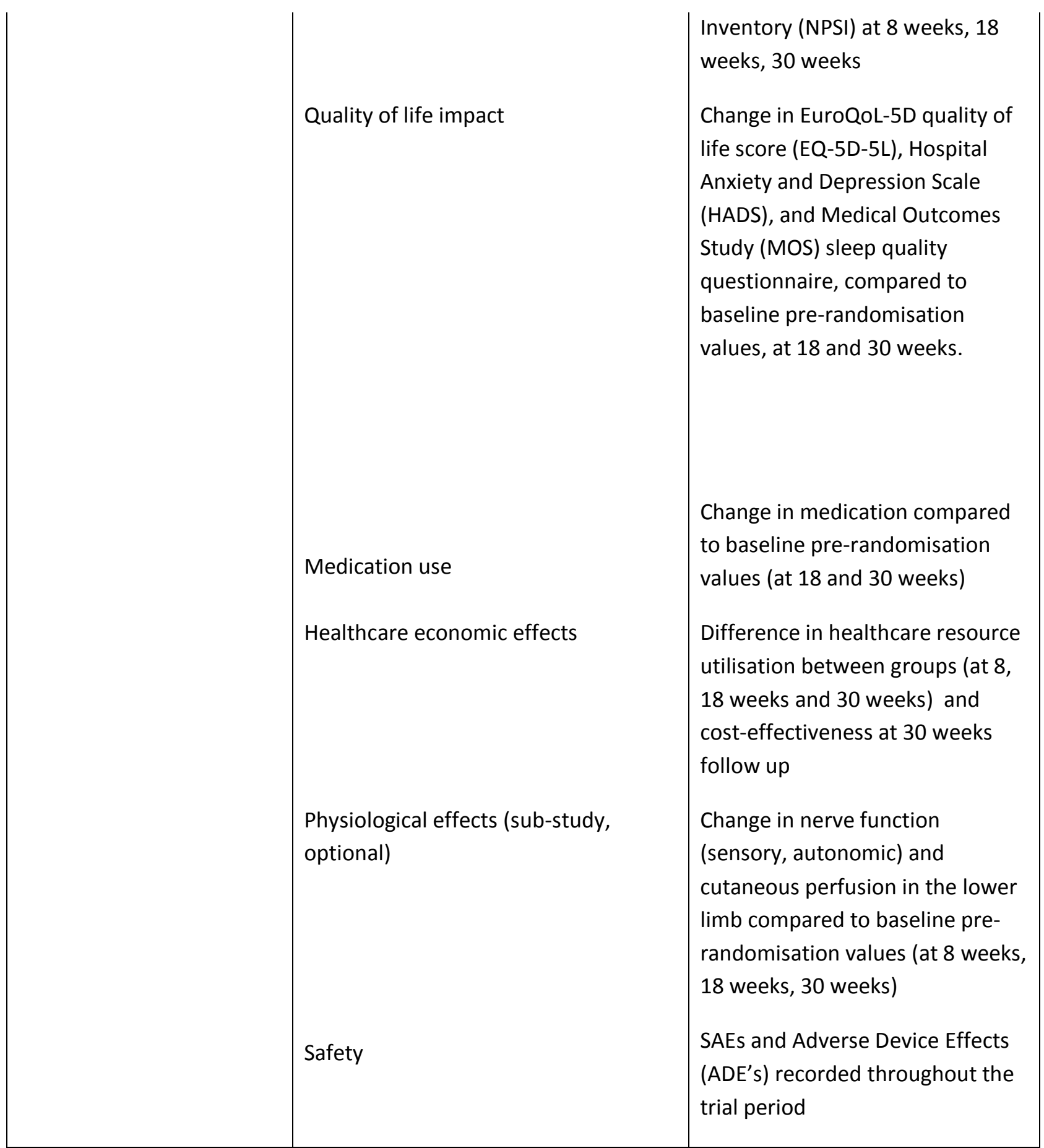

PENTAGONS: Diabetic Peripheral Neuropathy Treatment with Dorsal Root Ganglion Stimulation. 


\section{Af PENTAGONS}

\section{ABBREVIATIONS}

\begin{tabular}{|c|c|}
\hline ADE & Adverse Device Effects \\
\hline BPI & Brief Pain Inventory \\
\hline $\mathrm{Cl}$ & Chief Investigator \\
\hline CMM & Continued Medical Management \\
\hline CRF & Case Report Form \\
\hline CRPS & Complex Regional Pain Syndrome \\
\hline CSF & Cerebrospinal Fluid \\
\hline CTRG & Clinical Trials \& Research Governance, University of Oxford \\
\hline DRGS & Dorsal Root Ganglion Stimulation \\
\hline EQ-5D-5L & EuroQol quality of life questionnaire \\
\hline GCP & Good Clinical Practice \\
\hline GP & General Practitioner \\
\hline HADS & Hospital Anxiety and Depression Scale \\
\hline $\mathrm{HbA1c}$ & Glycosylated haemoglobin level \\
\hline HRA & Health Research Authority \\
\hline ICF & Informed Consent Form \\
\hline MDT & Multidisciplinary Team \\
\hline MNSI & Michigan Neuropathy Screening Instrument \\
\hline MOS & Medical Outcomes Study Sleep Survey \\
\hline NHS & National Health Service \\
\hline NHSFT & National Health Service Foundation Trust \\
\hline NPSI & Neuropathic Pain Symptom Inventory \\
\hline NRES & National Research Ethics Service \\
\hline PCS & Pain Catastrophising score \\
\hline PDN & Painful Diabetic Neuropathy \\
\hline $\mathrm{PI}$ & Principal Investigator \\
\hline PIS & Participant/ Patient Information Sheet \\
\hline PIC & Patient Identification Centre \\
\hline QST & Quantitative Sensory Testing \\
\hline$R \& D$ & NHS Trust R\&D Department \\
\hline REC & Research Ethics Committee \\
\hline
\end{tabular}

PENTAGONS: Diabetic Peripheral Neuropathy Treatment with Dorsal Root Ganglion Stimulation.

IRAS Project ID: 226499 REC Reference: 18/SC/0146

PENTAGONStrial_Protocol_V4.0_31Jul2019.docx31Jul2019

Clinical Research Protocol Template version 13.0

(C) Copyright: The University of Oxford and Oxford University Hospitals NHS Foundation Trust 2016 


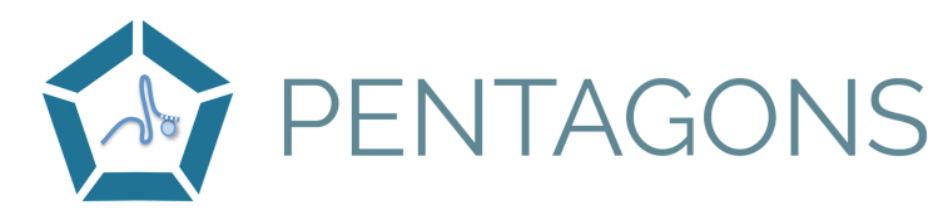

\begin{tabular}{|l|l|}
\hline SAE & Serious Adverse Event \\
\hline SADE & Serious Adverse Device Effect \\
\hline SUSAR & Suspected Unexpected Serious Adverse Reaction \\
\hline SCS & Spinal Cord Stimulation \\
\hline SOP & Standard Operating Procedure \\
\hline USADE & Unanticipated Serious Adverse Device Effect \\
\hline VAS & Visual Analogue Scale \\
\hline
\end{tabular}

PENTAGONS: Diabetic Peripheral Neuropathy Treatment with Dorsal Root Ganglion Stimulation.

IRAS Project ID: 226499 REC Reference: 18/SC/0146

PENTAGONStrial_Protocol_V4.0_31Jul2019.docx31Jul2019

Clinical Research Protocol Template version 13.0

(C) Copyright: The University of Oxford and Oxford University Hospitals NHS Foundation Trust 2016

Page 8 of 46 


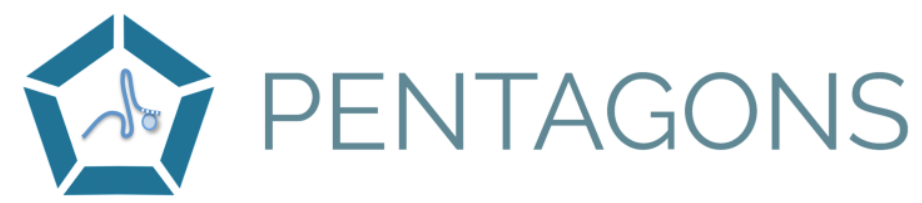

\section{BACKGROUND AND RATIONALE}

Painful diabetic peripheral neuropathy (PDN) is a common clinical problem. Approximately $50 \%$ of diabetics have peripheral neuropathy(1) and some 11-30\% experience neuropathic pain(2-4). The mainstays of pharmacological treatment are tricyclic antidepressants, selective serotonin reuptake inhibitors (SSRIs), anticonvulsants (gabapentin and pregabalin), and opiates (5-7). However there are a large number for whom pharmacotherapy is unsatisfactory either because it fails to achieve adequate pain relief or because the required dose of medication is such that it produces severe side effects (6).

Medically refractory neuropathic pain of various aetiologies may respond to electrical stimulation of sensory pathways, a method of treatment known as "neuromodulation". Implanted stimulator systems have been applied to the nervous system at all levels from cutaneous peripheral nerves to the cerebral cortex. Spinal cord stimulation (SCS) is the commonest such treatment. SCS involves the insertion of wires into the epidural space posterior to the spinal cord, either percutaneously through a needle or via a small surgical incision. Stimulation of the dorsal spinal cord through the wires produces a paraesthesia, typically described as a "buzzing" feeling, which has the effect of masking the pain. The level of the cord that is stimulated determines the area where the paraesthesia is felt. If a trial of stimulation through the wires produces beneficial analgesia the wires are then connected to an implantable pulse generator (IPG) which is sited subcutaneously. The IPG contains a battery together with electronics to generate stimulus pulses. The battery is surgically replaced every few years when depleted.

A standard metric of treatment effect in the literature is the percentage of patients experiencing a reduction of at least $50 \%$ in their pain score as measured by the visual analogue scale (VAS). In two recent randomised trials of SCS in patients with painful diabetic neuropathy (PDN), the proportion of patients achieving at least 50\% pain relief at 6 months was only $60 \%$ (8) and 59\% (9).

A new type of neuromodulation treatment, Dorsal Root Ganglion stimulation (DRGS), has been available since 2011. In DRGS, wires are inserted percutaneously through a needle into the epidural space, but rather than placing them over the back of the spinal cord as in SCS, an introducer sheath is used to steer the wire tip into the neural exit foramen so that the electrodes at the end of the wire lie over the dorsal root ganglion. This results in a number of advantages over SCS:

- The stimulation tends to be more focussed than with SCS. A broad area of stimulation can sometimes be desirable for widespread pain (for example affecting a whole limb) but more focussed stimulation is advantageous when the pain is localised to a more restricted area, such as the feet.

- Stimulation is not positional. With SCS, the electrodes are separated from the spinal cord by dura mater and a layer of cerebrospinal fluid (CSF) between the dura and the cord; the thickness of the CSF layer varies with posture with the result that stimulation efficacy is different when standing, sitting, or lying. There is no CSF between the dura and the DRG, so that the effect of DRGS is constant.

- With SCS, the CSF layer provides an electrical shunt meaning that much of the current being delivered by an SCS system is wasted, i.e. does not reach the cord. The absence of CSF over the ganglion results in much less current being required with DRGS. Typical DRGS currents are less

PENTAGONS: Diabetic Peripheral Neuropathy Treatment with Dorsal Root Ganglion Stimulation.

IRAS Project ID: 226499 REC Reference: 18/SC/0146

PENTAGONStrial_Protocol_V4.0_31Jul2019.docx31Jul2019

Clinical Research Protocol Template version 13.0

(C) Copyright: The University of Oxford and Oxford University Hospitals NHS Foundation Trust 2016

Page 9 of 46 


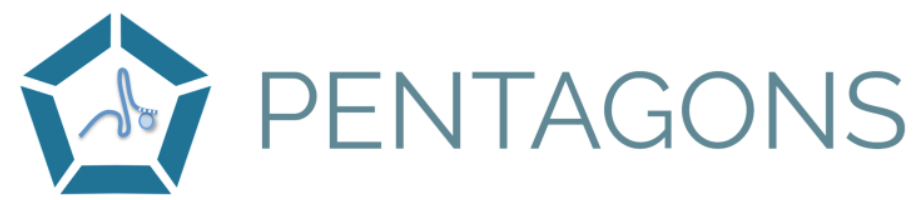

than one milliampere $(\mathrm{mA})$, whereas SCS usually requires several $\mathrm{mA}$. This means that stimulator batteries will last far longer between recharges or replacements.

The indications for which DRGS has been used in the lower limb include Complex Regional Pain Syndrome (CRPS), causalgia, phantom pain, post herniorrhaphy pain, testicular pain, and meralgia paraesthetica. DRGS appears to have a particular advantage for pain in the foot, which is frequently difficult to treat with SCS. In a large randomised trial directly comparing DRGS with SCS in patients with CRPS of the foot and causalgia of the lower limb (10), the proportion of patients obtaining at least $50 \%$ pain relief was $81 \%$ for DRGS versus $55 \%$ for SCS (intention to treat analysis; amongst the group who were actually implanted, the success rates were $93 \%$ and $72 \%$ respectively).

Another common and serious complication of diabetes is foot ulceration. A number of factors contribute to the genesis of ulcers including impaired sensation, reduced perfusion, and poor wound healing. Not uncommonly, persistent ulceration presages amputation. The treatment of diabetic ulcers and amputations costs the NHS approximately $f 650$ million per year (https://www.diabetes.org.uk/Documents/nhs-diabetes/footcare/footcare-for-people-with diabetes.pdf). SCS has been observed to cause peripheral vasodilatation (11). This is thought to be the result of the stimulation causing retrograde nerve impulses in sensory nerve fibres; these cause the nerve endings in the skin to release substances that dilate blood small blood vessels. This raises the possibility that DRGS may also improve perfusion, and in theory the stimulation site in DRGS should produce a superior effect to SCS. Evaluating the possible beneficial effects of DRGS on skin perfusion will be an important secondary aim of this study.

Diabetic neuropathy occurs because the diabetes damages small blood vessels that supply nerves. As well as pain, it causes impairment of sensation and loss of autonomic function. It is possible that if DRGS improves skin perfusion, it might also improve nerve perfusion and function. We will therefore also quantify these deficits and see what effect, if any, DRGS has on them.

Population to be studied: Patients at the study centres with medication-resistant severe painful diabetic neuropathy affecting the feet, who are deemed suitable for the procedure by the multi-disciplinary team. The results of the project will be generalisable to similar patients elsewhere.

The intervention: DRGS as described above. The device is CE marked and it will be used on-label.

\section{Main research questions:}

- How effective is DRGS as a treatment for PDN?

- Does DRGS improve foot perfusion in patients with PDN?

\section{Potential risks of DRGS implantation:}

The procedure requires the use of X-rays to help guide the stimulator wires into position. Because $\mathrm{X}$-rays are potentially harmful to a developing baby, this procedure cannot be carried out if there is any chance that the patient may be pregnant.

PENTAGONS: Diabetic Peripheral Neuropathy Treatment with Dorsal Root Ganglion Stimulation.

IRAS Project ID: 226499 REC Reference: 18/SC/0146

PENTAGONStrial_Protocol_V4.0_31Jul2019.docx31Jul2019

Clinical Research Protocol Template version 13.0

(C) Copyright: The University of Oxford and Oxford University Hospitals NHS Foundation Trust 2016

Page 10 of 46 


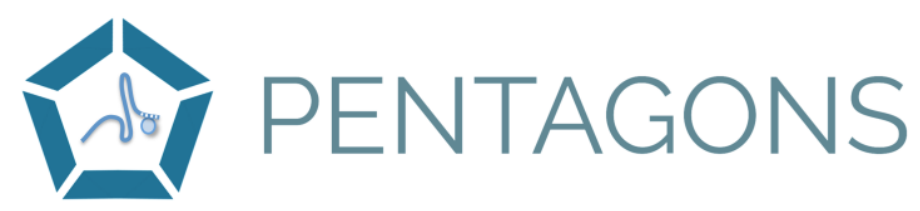

Device infection: In people without diabetes who are having similar stimulator implants for other types of pain, the risk of an infection of the stimulator system is approximately 1 person in every 25 . In diabetes, the extra sugar in the bloodstream may make infection up to twice as likely. If the stimulator system gets infected it usually has to be removed as devices that are colonised cannot in general be sterilised in situ with antibiotic treatment. If it was very effective before it was removed, then it can be replaced a few months later once the infection has cleared completely. Some studies have suggested a link between poor preoperative diabetic control and increased infection rates following implant surgery but this is debated and other studies failed to find such an association. We will record and monitor infection rates as described in section (9).

- Lead migration: The percutaneously inserted DRG wires may move slightly such that they are no longer in good contact with the DRG and any pain relieving benefit is lost. This may occur at any time after implantation but is most likely in the first few weeks. The chance of this happening with DRGS is not exactly known, but may be up to 1 in 5 . When it occurs, it is standard practice to offer lead replacement in order to regain effect.

- Cerebrospinal fluid (CSF) leakage (less than 1 in 100): The nerves in the spine are contained within a sheath, and within that sheath they are surrounded by a watery fluid called CSF. The stimulator wires are positioned outside that sheath and the electrical pulses pass through the sheath to get to the DRG. There is a risk of a leak of the CSF through the sheath, and this can cause a headache after the operation. Usually this settles down within a few days without intervention, but occasionally it persists. If so, it can be treated with an injection in the back to seal up the leak, in a procedure known as blood patching.

- Nerve damage (less than 1 in 100): This can be due to direct injury as the lead is being inserted, or to bleeding around the nerves.

- Other risks (less than 1 in 100): This includes deep vein thrombosis.

There are no specific risks associated with the additional assessments in this trial (including the physiology sub-study; see Appendix A).

PENTAGONS: Diabetic Peripheral Neuropathy Treatment with Dorsal Root Ganglion Stimulation.

IRAS Project ID: 226499 REC Reference: 18/SC/0146

PENTAGONStrial_Protocol_V4.0_31Jul2019.docx31Jul2019

Clinical Research Protocol Template version 13.0

(C) Copyright: The University of Oxford and Oxford University Hospitals NHS Foundation Trust 2016

Page 11 of 46 


\section{Af PENTAGONS}

\section{OBJECTIVES AND OUTCOME MEASURES}

\begin{tabular}{|c|c|c|}
\hline Objectives & Outcome Measures & $\begin{array}{l}\text { Timepoint(s) of } \\
\text { evaluation of this } \\
\text { outcome measure } \\
\text { (if applicable) }\end{array}$ \\
\hline $\begin{array}{l}\text { Primary Objective } \\
\text { To establish the efficacy of Dorsal } \\
\text { Root Ganglion Stimulation (DRGS) } \\
\text { for pain relief in Painful Diabetic } \\
\text { Neuropathy of the feet. }\end{array}$ & $\begin{array}{l}\text { Percentage of participants with a } 50 \% \text { or } \\
\text { more reduction in their pain VAS score } \\
\text { compared to baseline pre-randomisation } \\
\text { values }\end{array}$ & 30 weeks \\
\hline Secondary Objectives & & \\
\hline As above & $\begin{array}{l}\text { Pain VAS scores } \\
\text { Brief Pain Inventory (BPI) and Neuropathic } \\
\text { Pain Symptom Inventory (NPSI) }\end{array}$ & $\begin{array}{l}\text { 8, and } 18 \text { weeks } \\
8,18 \text { and } 30 \text { weeks }\end{array}$ \\
\hline Quality of life impact & $\begin{array}{l}\text { Change in EQ5D } \\
\text { HADS and MOS }\end{array}$ & $\begin{array}{l}18 \text { and } 30 \text { weeks } \\
18 \text { and } 30 \text { weeks }\end{array}$ \\
\hline Medication use & Change in medication & 18 and 30 weeks \\
\hline Healthcare economic effects & $\begin{array}{l}\text { Difference in healthcare resource } \\
\text { utilisation* between groups }\end{array}$ & 8,18 and 30 weeks \\
\hline $\begin{array}{l}\text { Physiological effects (sub-study, } \\
\text { optional) }\end{array}$ & $\begin{array}{l}\text { Change in nerve function (sensory, } \\
\text { autonomic) and cutaneous perfusion in } \\
\text { the lower limb. }\end{array}$ & 8,18 and 30 weeks \\
\hline Safety & SAEs and Adverse device effects reported & $\begin{array}{l}\text { Throughout } \\
\text { trial period }\end{array}$ \\
\hline
\end{tabular}

* Diaries will capture details of contact with health or social care professionals, medication use, and the patient's day by day ability to do their usual activities. Non responders will be followed up with a phone call reminder to return the questionnaire. This health resource use information will be supplemented by procedure, complications, ADE's and SAE's and follow-up data documented in the CRF's.

PENTAGONS: Diabetic Peripheral Neuropathy Treatment with Dorsal Root Ganglion Stimulation.

IRAS Project ID: 226499 REC Reference: 18/SC/0146

PENTAGONStrial_Protocol_V4.0_31Jul2019.docx31Jul2019

Clinical Research Protocol Template version 13.0

(C) Copyright: The University of Oxford and Oxford University Hospitals NHS Foundation Trust 2016 


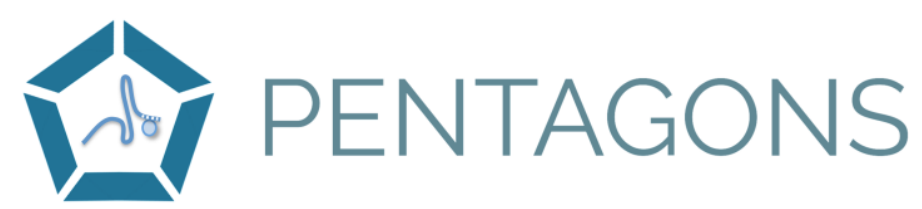

\section{STUDY DESIGN}

This is an open label multi-centre randomised trial of DRGS versus continued medical management (CMM).

Patients will be followed up as part of the study for a period of 30 weeks from randomisation.

Identification will take place in the diabetic clinic, podiatry clinic, orthotic clinic, vascular surgery clinic, specialist pain clinic, specialist neuromodulation clinic and Patient Identification Centres (PICS) (see section 7.1), where a PIS and or a participant invitation letter will be provided (see section 7.2).

Study visits will be as follows:

- All potential participants:

- Initial outpatient consultation in neuromodulation clinic at which time patients who wish to participate will be enrolled

- Detailed screening to ensure eligibility (section 7.3) including multidisciplinary team (MDT) discussion as per routine clinical practice to confirm suitability for treatment

- All eligible participants (meet inclusion and exclusion criteria):

- Baseline assessment in outpatient clinic (see section 7.4) and randomisation (see section 7.5)

- $\quad$ DRGS arm only (post-randomisation):

- Preoperative assessment in outpatient clinic (i.e. health check that is done routinely in preparation for DRGS) including an MRI scan (performed during the last year or at this assessment) and ECG to confirm the participant's eligibility to receive DRGS

- Inpatient DRGS procedure, switch-on, titration of stimulation expected at approximately six weeks post randomisation

- Outpatient visit at two weeks postoperative visit to check system function and adjust if necessary

All participants will be asked to complete pain and QoL questionnaires at the following time points:

- Follow-up time point 1:

- $\mathrm{CMM}$ arm -8 weeks post randomisation

- DRGS arm - 2 weeks post DRGS implantation (it is anticipated that this time point will be $\sim 8$ weeks post randomisation)

- Follow-up time point 2

- 18 weeks post randomisation

- Follow-up time point 3

- 30 weeks post randomisation

Participants will also be provided with a resource utilisation diary to be completed over three time periods for the duration of the trial. Diary 1 ( 8 week duration - Randomisation to 8 weeks), Diary 2 (10 week duration - 8-18 weeks) and Diary 3 (12 week duration - 18-30 weeks). Please refer to Section 7 and Appendices $B$ and $C$ for full details on study visits and study flowchart.

PENTAGONS: Diabetic Peripheral Neuropathy Treatment with Dorsal Root Ganglion Stimulation.

IRAS Project ID: 226499 REC Reference: 18/SC/0146

PENTAGONStrial_Protocol_V4.0_31Jul2019.docx31Jul2019

Clinical Research Protocol Template version 13.0

(C) Copyright: The University of Oxford and Oxford University Hospitals NHS Foundation Trust 2016

Page 13 of 46 


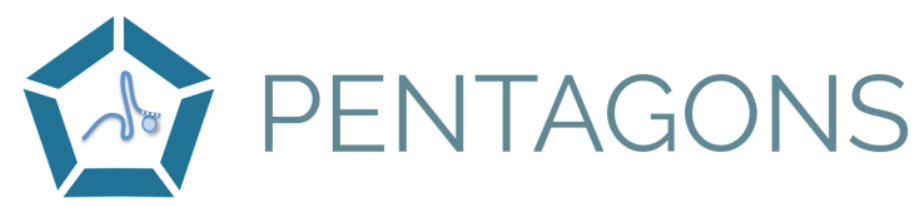

At the lead site, a subset of participants in both arms will undergo Quantitative Sensory Testing (QST) and tests of autonomic function, blood flow and perfusion, as part of a separate sub study (see Appendix A). This testing will be performed using standard electronic equipment specifically designed for these purposes and approved for use in human subjects. All participants, at the lead site, in both arms, will be invited to take part. A separate informed consent will be obtained for this on a dedicated ICF.

\section{PARTICIPANT IDENTIFICATION}

\subsection{Study Participants}

Participants with PDN of the feet measuring at least $50 \mathrm{~mm}$ on a $0-100 \mathrm{~mm}$ VAS on an average day, for at least 6 months despite appropriate medical management including trials of specified analgesic medications.

\subsection{Inclusion Criteria}

The participant may enter the trial if ALL of the following apply:

- Participant is willing and able to give informed consent for participation in the trial.

- Male or Female, aged 18 years or above.

- Diagnosed with PDN of the feet, present for at least 6 months and scoring at least $50 \mathrm{~mm}$ on a pain VAS despite medical treatment.

- Score of at least 2 out of 10 using the Michigan Neuropathy Screening Instrument for peripheral neuropathy (see Appendix D)

- Participant has tried at least two first-line pharmacotherapy options and either is refractory to painkilling medication, or the required dose of medication to alleviate pain causes severe side effects. These should include at least one antiepileptic (gabapentin or pregabalin), and at least one antidepressant (amitriptyline or duloxetine).

- Stable analgesic medication regimen for at least 4 weeks prior to trial entry. This includes patients not taking analgesics at all (either because they are refractory, or because side effects are too severe).

- Female participants of child bearing potential must be willing to either state prior to enrolment that there is no chance that they could be pregnant, or if there is a chance that they might be pregnant, to undergo a pregnancy test. This is because the insertion of a DRGS system requires $X$ ray fluoroscopy that might be harmful to a developing foetus.

- If, in the Investigator's opinion, the patient is able and willing to comply with all trial requirements.

- If, in the opinion of a consultant anaesthetist after review of case notes and medical history, there are no contraindications to DRGS surgery

- Willing to allow his or her General Practitioner and consultant, if appropriate, to be notified of participation in the trial.

PENTAGONS: Diabetic Peripheral Neuropathy Treatment with Dorsal Root Ganglion Stimulation.

IRAS Project ID: 226499 REC Reference: 18/SC/0146

PENTAGONStrial_Protocol_V4.0_31Jul2019.docx31Jul2019

Clinical Research Protocol Template version 13.0

(C) Copyright: The University of Oxford and Oxford University Hospitals NHS Foundation Trust 2016

Page 14 of 46 


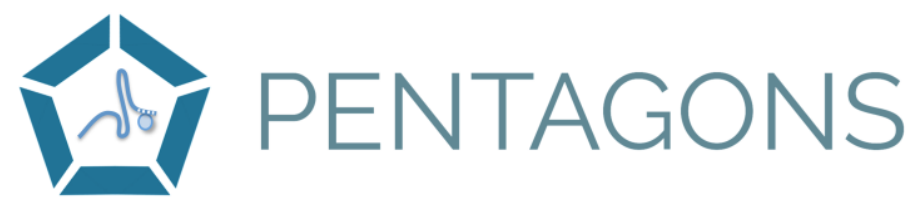

\subsection{Exclusion Criteria}

The participant may not enter the trial if ANY of the following apply:

- Female participant who is pregnant or planning pregnancy during the course of the trial.

- Lack of good understanding of both written and spoken English. Most of the outcome measures, including the primary outcome measure, are in the form of questionnaires and these require good understanding of written English in order to provide valid measures. Fluent spoken English is needed during the implantation procedure when the participant is asked to confirm that the stimulation effect can be felt in the painful area.

- Active infection other than viral infections that are mild and confidently expected to be selflimiting within two weeks (e.g. common colds).

- Anticoagulant treatment or other coagulopathy.

- Diabetic ulcers at the time of, or in the 3 months prior to, recruitment.

- Participant rates their pain consistently as 10 out of 10 , even on their best day. This indicates a failure to understand the principles of VAS use, or pain catastrophisation, either of which will confound pain scoring.

- Significant pain elsewhere in the body from any condition, which the participant is not easily able to rate separately from their foot pain. Participants must be able to clearly rate their foot pain alone and will be excluded if in the opinion of the Investigator they are unable to do so.

- Any other significant disease or disorder which, in the opinion of the Investigator, may either put the participant at risk because of participation in the trial, or may influence the result of the trial, or the participant's ability to participate in the trial.

- Opiate use greater than the maximum recommended amount for non-cancer pain $(120 \mathrm{mg}$ morphine or equivalent per 24 hours).

- Participation in a clinical trial of an investigational medicinal product (CTIMP) in the past 12 weeks.

- Previous lumbar spine surgery at or below the level of the third lumbar vertebra (L3). This is because the scarring following lumbar spine surgery frequently makes it impossible to pass a DRG wire.

- Previous failed Spinal Cord Stimulation therapy or other neuromodulation treatment

- Previous lumbar or sacral radiofrequency nerve lesioning

- Inability to have a preoperative MRI scan, either due to claustrophobia or due to the presence of a contraindication to MRI scanning.

- A previously performed MRI scan shows a contraindication to DRG lead insertion (if previous MRI scans are available they should be reviewed at this stage, prior to randomisation, to confirm no contraindication is present)

- Body Mass Index (BMI) $>30$

- Patient is undergoing psychological therapy

\section{STUDY PROCEDURES}

PENTAGONS: Diabetic Peripheral Neuropathy Treatment with Dorsal Root Ganglion Stimulation.

IRAS Project ID: 226499 REC Reference: 18/SC/0146

PENTAGONStrial_Protocol_V4.0_31Jul2019.docx31Jul2019

Clinical Research Protocol Template version 13.0

(C) Copyright: The University of Oxford and Oxford University Hospitals NHS Foundation Trust 2016

Page 15 of 46 


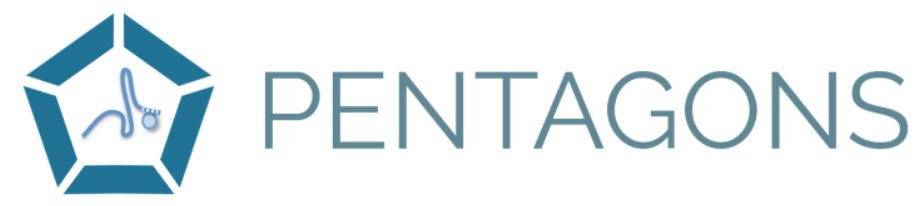

\subsection{Recruitment}

Potential participants will be identified from attendees at a diabetic clinic, podiatry clinic, orthotic clinic, vascular surgery clinic, specialist pain clinic, or specialist neuromodulation clinic. T

They will be initially approached by nursing or medical staff who will provide a PIS and/or participant invitation letter to those who wish to find out more about the study. Verbal permission will be sought (with a note inserted into the medical records) for a member of the study team to contact them by telephone or face to face to discuss the study further and to give the participant the opportunity to ask any questions.

Potential participants will also be identified from PIC sites, either hospital diabetic clinics within the following CRN's or PIC sites within primary care, using their databases to screen against the inclusion/exclusion criteria. (It is understood that not all of the criteria will exist as searchable fields in such databases, and the expectation will be that as full a set of criteria as is practical will be used, with screening using remaining criteria then being completed when potential participants attend the neuromodulation clinic.) A participant invitation letter accompanied by a PIS will be posted out by the individual carrying out the search. It will provide contact details for a member of the study team whom they can contact to ask any questions and to arrange an appointment in person to discuss the study further. The PICs will include: CRN: Thames Valley and South Midlands, CRN Greater Manchester, CRN: North East and North Cumbria, CRN: Wessex, CRN: South London and CRN: West Midlands.

To further maximise recruitment, posters will be displayed in the aforementioned clinics and the PIS uploaded to the Diabetes UK website.

We will also utilise the database for the Pain in Neuropathy Study (PINS) run by Prof D Bennett at the University of Oxford, which includes details of participants in that study who indicated that they are interested in hearing about other studies for which they may be eligible, and provided consent to be contacted for such purposes.

Patients who are interested in taking part will be assessed in the routine way for potential neuromodulation patients, which includes a neuromodulation clinic appointment with the $\mathrm{PI}$ or CoInvestigator and RN, where the trial will be discussed in depth. The potential participant will be informed that if they are allocated DRGS in the trial, they will need to undergo a pre-op assessment that includes an MRI scan (unless they have had one within the previous 12 months that satisfies the criteria in the TSI) and ECG, to confirm they are eligible to receive DRGS. Consent for the MRI scan and ECG forms part of the trial consent form.

\subsection{Informed Consent}

The participant must personally sign and date the latest approved version of the Informed Consent Form (ICF) before any study-specific procedures are performed (including MRI scan and ECG). The person who

PENTAGONS: Diabetic Peripheral Neuropathy Treatment with Dorsal Root Ganglion Stimulation.

IRAS Project ID: 226499 REC Reference: 18/SC/0146

PENTAGONStrial_Protocol_V4.0_31Jul2019.docx31Jul2019

Clinical Research Protocol Template version 13.0

(C) Copyright: The University of Oxford and Oxford University Hospitals NHS Foundation Trust 2016

Page 16 of 46 


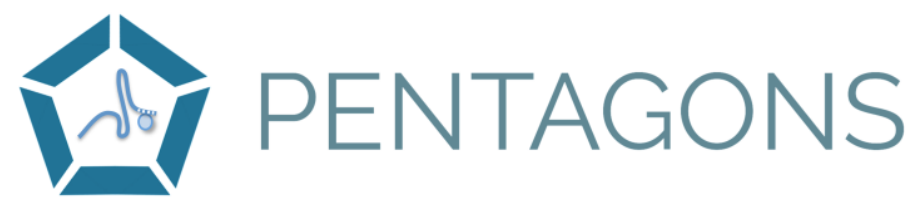

obtained consent must be suitably qualified and experienced, and have been authorised to do so by the Chief/Principal Investigator and detailed on the delegation log. A copy of the signed ICF will be given to the participant and one copy will be placed in the participant's medical notes. The original signed ICF will be retained in the Investigator Site File (ISF).

The PIS will detail no less than: the exact nature of the study; what it will involve for the patient; the implications and constraints of the protocol; the requirement that an MRI scan and ECG must take place if the participant is allocated DRGS (and that these tests may show the participant is ineligible for DRGS, in which case they will receive standard NHS treatment for their condition (CMM); and be followed up in accordance with the protocol), known side effects and any risks involved in taking part. It will be clearly stated that the patient is free to withdraw from the study at any time for any reason without prejudice to future care, without affecting their legal rights, and with no obligation to give the reason for withdrawal. The participant will also be requested to consent to provide their e-mail address, mobile phone number and home address to enable remote electronic completion of pain and QoL questionnaires and paper completion of the health resource use diaries. If the participant does not wish to consent to electronic completion of the pain and QoL questionnaires, these can be completed on paper and will be posted by the PENTAGONS Trial Office.

The patient will be allowed as much time as wished to consider the information, and the opportunity to question the Investigator, their GP or other independent parties to decide whether they will participate in the study.

The physiology sub-study (see Appendix A) has a separate PIS and ICF. Participants who are invited to join the sub study will be provided with the sub-study PIS and separate consent will be obtained for this, following the same principles as for the main study.

\subsection{Screening and Eligibility Assessment}

Maximum duration allowed between screening and randomisation: 12 weeks.

Screening will involve:

- Demographics: Must be at least 18 years old

- Use of the validated Michigan Neuropathy Screening Instrument (MNSI) to confirm the presence of neuropathy (indicated by a score of at least 2 out of 10) (see Appendix D)

- Check against inclusion criteria including the location, severity, and duration of neuropathic pain, and current and previously tried analgesic medication (see section 6.2 for full details)

- Check against exclusion criteria including history of lower lumbar spine surgery, anticoagulant medication or known coagulopathy, current or recent ulceration or signs of infection (see section 6.3 for full details)

- Case note review by a consultant anaesthetist to ensure that there are no obvious contraindications or significant concerns regarding fitness for the surgical procedure under local anaesthesia and sedation. This will prevent or minimise post-randomisation exclusions at the time of formal preoperative checks for surgical patients.

PENTAGONS: Diabetic Peripheral Neuropathy Treatment with Dorsal Root Ganglion Stimulation.

IRAS Project ID: 226499 REC Reference: 18/SC/0146

PENTAGONStrial_Protocol_V4.0_31Jul2019.docx31Jul2019

Clinical Research Protocol Template version 13.0

(C) Copyright: The University of Oxford and Oxford University Hospitals NHS Foundation Trust 2016

Page 17 of 46 


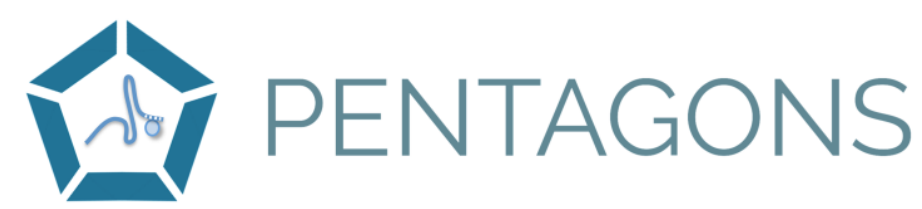

- Discussion at the neuromodulation multidisciplinary team (MDT) meeting. This is a standard part of the normal process for all neuromodulation patients.

\subsection{Baseline Assessments}

The following Baseline Assessments will be undertaken:

- Demographics

- Relevant medical history

- Diabetes history

- Current analgesic medication

Results of these assessments will be recorded first in the medical notes and then on a Baseline Case Report Form (CRF).

Participants will be asked to complete the following pain and QoL of life questionnaires:-

- Pain scoring:

O VAS

- Brief Pain Inventory (BPI)

- Neuropathic pain symptom inventory (NPSI)

- Pain catastrophising scale (PCS)

- Quality of life (QoL) scores:

O Euroqol questionnaire (EQ-5D-5L)

- Hospital anxiety and depression scale (HADS)

- MOS Sleep Survey (MOS)

These will be completed in clinic by the participant online using a tablet computer; provided by the PENTAGONS trial office. Paper versions will be available as a backup which if used will be posted to the central PENTAGONS trial office.

\subsection{Randomisation, blinding and code-breaking}

It is not possible to blind the patients, surgeons, or research staff in this trial. Therefore after randomisation there will be no requirement for code-breaking.

Baseline assessments will be pre-randomisation.

Patients will be randomised 1:1 to DRGS or continued medical management (CMM) using a computerbased randomisation system (RRAMP) provided by the Oxford Clinical Trials Research Unit (OCTRU). Randomisation will be stratified by centre. The statistician will generate the allocation sequence using variable block sizes and this will be embedded into the computer system so that treatment allocation is not predictable

PENTAGONS: Diabetic Peripheral Neuropathy Treatment with Dorsal Root Ganglion Stimulation.

IRAS Project ID: 226499 REC Reference: 18/SC/0146

PENTAGONStrial_Protocol_V4.0_31Jul2019.docx31Jul2019

Clinical Research Protocol Template version 13.0

(C) Copyright: The University of Oxford and Oxford University Hospitals NHS Foundation Trust 2016 


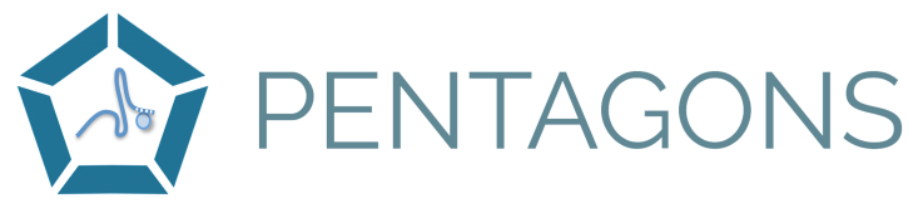

\subsection{Treatment Schedule}

\section{DRGS arm}

In the DRGS arm, following randomisation and before surgery there will be a routine preoperative assessment for surgical safety purposes. This will include blood tests, and an electrocardiogram (ECG). Participants will also undergo a lumbar spine MRI scan. This is a standard procedure done in order to check that there is adequate room in the space around the DRG for safe electrode insertion. Female patients of childbearing potential will undergo a pregnancy test and if found to be pregnant will not be able to undergo DRGS because of the need to use X-rays during the procedure.

If any of these preoperative assessments shows that the participant is unable to receive DRGS, the participant will continue to receive CMM. They will be followed up at the same time points as if they had received DRGS and will be analysed as part of the DRGS arm (intention to treat analysis).

A Trial Specific Instruction (TSI) outlining the process for reviewing the MRI will be provided to all sites.

The surgical episode in the DRGS arm will be as close as possible to 6 weeks after the baseline assessment and randomisation. The surgical intervention is described in section 8 .

\section{CMM arm}

Patients in the CMM arm will continue on their current medication. The study team will not alter their medication during the course of the trial. Other physicians including their GP or other specialists will not be prevented from making medication changes, however it is unlikely that changes will occur during the study period because patients will have tried all suitable options before being considered for DRGS (minimum requirements for medications tried are stated in the inclusion criteria).

\subsection{Subsequent Visits and assessments}

Following randomisation participants in BOTH arms will be given their FIRST health resource use diary and requested to start completing it immediately. They will be asked to continue completing it until the SECOND diary is issued (about 8 weeks post randomisation) A pre-paid envelope will be provided so that the participant can post the FIRST resource use diary to the PENTAGONS Trial Office

DRGS Arm: Participants allocated DRGS will be followed up at the following time points:

- DRGS procedure (approximately 6 weeks post-randomisation)

Participants will receive the DRGS intervention and initial programming.

- 2 weeks post-surgery (it is anticipated that this time point will be 8 weeks post randomisation)

Participants will be seen in an outpatient clinic for a routine postoperative review.

PENTAGONS: Diabetic Peripheral Neuropathy Treatment with Dorsal Root Ganglion Stimulation.

IRAS Project ID: 226499 REC Reference: 18/SC/0146

PENTAGONStrial_Protocol_V4.0_31Jul2019.docx31Jul2019

Clinical Research Protocol Template version 13.0

(C) Copyright: The University of Oxford and Oxford University Hospitals NHS Foundation Trust 2016

Page 19 of 46 


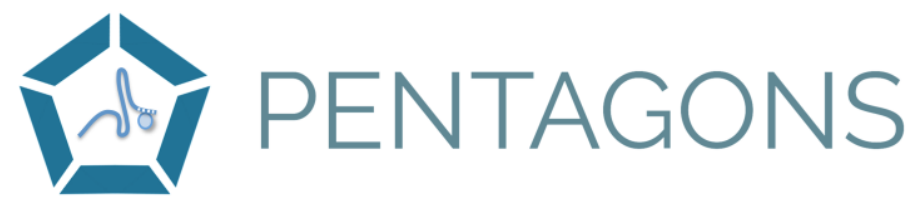

Participants will also be contacted by the PENTAGONS Trial Office and requested to complete VAS, BPI, NPSI and EQ-5D-5L questionnaires on-line. Paper versions are also available for those who do not want to complete them electronically, and will be posted out by the PENTAGONS Trial Office to complete by hand, along with a pre-paid envelope for return.

Also, using the pre-paid envelope, previously provided, participants will be asked to post back their FIRST health resource use diary; to the PENTAGONS Trial Office. They will also be sent, by post, their SECOND health resource use diary, and requested to complete it for the next 10 weeks. A pre-paid envelope will be provided for return.

- 18 weeks post-randomisation

Participants will be contacted by the PENTAGONS Trial Office and requested to complete VAS, BPI, NPSI, EQ-5D, HADS and MOS questionnaires on-line. Paper versions are also available for those who do not want to complete them electronically, and will be posted out by the PENTAGONS Trial Office to complete by hand, along with a pre-paid envelope for return.

Also, using the pre-paid envelope, previously provided, participants will be asked to post back their SECOND health resource use diary; to the PENTAGONS Trial Office. They will also be sent, by post, their THIRD health resource use diary, and requested to complete it for the next 12 weeks. A prepaid envelope will be provided for return.

- 30 weeks post-randomisation

Participants will be contacted by the PENTAGONS Trial Office and requested to complete VAS, BPI, NPSI, EQ-5D, HADS and MOS questionnaires on-line. Paper versions are also available for those who do not want to complete them electronically, and will be posted out by the PENTAGONS Trial Office to complete by hand, along with a pre-paid envelope for return.

Also, using the pre-paid envelope, previously provided, participants will be asked to post back their THIRD health resource use diary to the PENTAGONS Trial Office. -

CMM Arm: Participants allocated CMM will be followed up at the following time points:

- 8 weeks post- randomisation

Participants will be contacted by the PENTAGONS Trial Office and requested to complete VAS, BPI, NPSI and EQ-5D questionnaires on-line. Paper versions are also available for those who do not want to complete them electronically, and will be posted out by the PENTAGONS Trial Office to complete by hand, along with a pre-paid envelope for return.

Also, using the pre-paid envelope, previously provided, participants will be asked to post back their FIRST health resource use diary; to the PENTAGONS Trial Office. They will also be sent, by post, their SECOND health resource use diary, and requested to complete it for the next 10 weeks. A pre-paid envelope will be provided for return.

- 18 weeks post-randomisation

Participants will be contacted by the PENTAGONS Trial Office and requested to complete VAS, BPI, NPSI, EQ-5D, HADS and MOS questionnaires on-line. Paper versions are also available for those

PENTAGONS: Diabetic Peripheral Neuropathy Treatment with Dorsal Root Ganglion Stimulation.

IRAS Project ID: 226499 REC Reference: 18/SC/0146

PENTAGONStrial_Protocol_V4.0_31Jul2019.docx31Jul2019

Clinical Research Protocol Template version 13.0

(C) Copyright: The University of Oxford and Oxford University Hospitals NHS Foundation Trust 2016

Page 20 of 46 


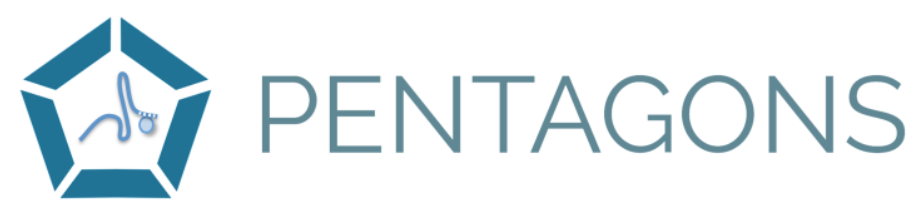

who do not want to complete them electronically, and will be posted out by the PENTAGONS Trial Office to complete by hand, along with a pre-paid envelope for return.

Also, using the pre-paid envelope, previously provided, participants will be asked to post back their SECOND health resource use diary; to the PENTAGONS Trial Office. They will also be sent, by post, their THIRD health resource use diary, and requested to complete it for the next 12 weeks. A prepaid envelope will be provided for return.

- 30 weeks post-randomisation

Participants will be contacted by the PENTAGONS Trial Office at 30 weeks post-randomisation and asked to complete VAS, BPI, NPSI, EQ-5D, HADS and MOS questionnaires on-line. Paper versions are also available for those who do not want to complete them electronically, and will be posted out by the PENTAGONS Trial Office to complete by hand, along with a pre-paid envelope for return.

Also, using the pre-paid envelope, previously provided, participants will be asked to post back their THIRD health resource use diary.

\subsection{Withdrawal from the Randomised Intervention and Withdrawal from Follow-Up}

Participants may withdraw consent from undergoing or continuing with their randomised intervention at any time. Their decision will not affect the standard of care the participant receives.

Members of the trial team will explain to participants the value of continuing to complete questionnaires at follow-up. Where possible, participants who have withdrawn from the trial intervention should continue to be followed up, unless they withdraw consent for this. Reduced follow-up will be possible for participants who are unwilling to complete the full follow-up questionnaires and diaries.

In the event of withdrawal from the randomised intervention, or if participants are unable to undergo the DRGS procedure due to contraindications, participants will be treated as per routine practice.

\section{Loss to follow-up}

If a participant is lost to follow-up at a site every effort should be made to contact the participant's GP to obtain information on the participant's status. If a follow-up is missed because of non-response to 3 contact attempts by the study team, subsequent follow-up assessments should still be performed.

\section{Management in the event of withdrawal}

Patients who withdraw from the study after having had DRGS will be followed up by the neuromodulation team as per routine practice.

Patients who withdraw from the study who have not had DRGS will be referred back to their GP and diabetologist for ongoing care. They may be considered for DRGS outside the study after study completion, if the study shows that DRGS is effective for this indication, via the usual NHS pathway.

The reason for the withdrawal will be recorded on the Discontinuation CRF and in the medical notes.

PENTAGONS: Diabetic Peripheral Neuropathy Treatment with Dorsal Root Ganglion Stimulation.

IRAS Project ID: 226499 REC Reference: 18/SC/0146

PENTAGONStrial_Protocol_V4.0_31Jul2019.docx31Jul2019

Clinical Research Protocol Template version 13.0

(C) Copyright: The University of Oxford and Oxford University Hospitals NHS Foundation Trust 2016

Page 21 of 46 


\section{金 PENTAGONS}

\subsection{Definition of End of Study}

The end of the trial is once all data has been collected and queries resolved following the last visit of the last participant.

\subsection{Treatment post completion of the Study}

Post-study treatment will be as follows:

Patients in the DRGS arm will be followed up by the neuromodulation team as per routine practice:

- If DRGS was effective for the patient then system maintenance (reprogramming and battery replacements as and when necessary) will be provided by the neuromodulation team on an indefinite basis

- If DRGS was ineffective for the patient then the patient will be offered system removal. This is a straightforward procedure with minimal risk, lasting approximately 15 minutes. The incision over the IPG only is opened, and this allows removal of the IPG and withdrawal of the stimulator wires from the same site. This is done under local anaesthetic and no further $\mathrm{X}$-rays are required.

Patients in the CMM arm:

- If the study suggests that DRGS is effective for this indication, patients in the CMM arm will be offered DRGS immediately after study completion. Further aftercare from that point will be the same as for patients in the DRGS arm (see above).

- If the study suggests that DRGS is ineffective for this indication, patients in the CMM arm will not be offered DRGS after study completion.

\subsection{Sample Handling}

Blood samples (maximum 20ml) are taken on a single occasion at the preoperative assessment (patients undergoing DRGS only) in order to perform routine preoperative health check tests. These are not used for any other research purpose. Samples are sent straight to the hospital laboratory where they are tested and they are destroyed by the hospital laboratory after testing. The direct care team will have access to the results of the tests for preoperative health check purposes only.

Preoperative tests include a Full Blood Count, Urea and Electrolytes, and Coagulation screen, and HbA1c.

\section{INTERVENTIONS}

\subsection{Surgical procedure}

PENTAGONS: Diabetic Peripheral Neuropathy Treatment with Dorsal Root Ganglion Stimulation.

IRAS Project ID: 226499 REC Reference: 18/SC/0146

PENTAGONStrial_Protocol_V4.0_31Jul2019.docx31Jul2019

Clinical Research Protocol Template version 13.0

(C) Copyright: The University of Oxford and Oxford University Hospitals NHS Foundation Trust 2016

Page 22 of 46 


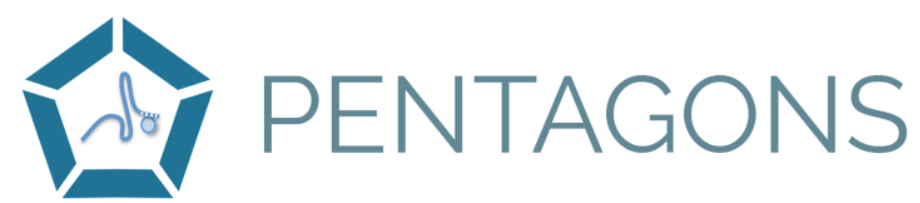

The intervention is the surgical implantation of a DRGS system.

This is a CE marked device and is being used on-label without any modification.

The first step in the procedure is to pass a fine wire through a needle into the spine. This is similar to having an epidural anaesthetic and is done under local anaesthetic and sedation. The wire is guided using fluoroscopy (X-rays) to lie over the selected DRG. Up to four wires can be sited, in order to stimulate up to four DRGs. It is anticipated that in this study most participants would require two wires (one at the L5 level on each side). Once in position, sedation is stopped and the wires are tested using an external stimulator to generate paraesthesia (a tingling or buzzing feeling) in the area of pain. If at this stage it is not possible to produce stimulation that covers the painful area then the wires are removed and the procedure abandoned.

Once the effect has been confirmed the sedation is restarted and an implantable pulse generator (IPG, a device resembling a heart pacemaker that contains a battery and electronics to produce stimulus pulses) is implanted under the skin and the wires connected to it.

The device is switched on and stimulus levels titrated against the patient's pain, and they are then discharged. They are reviewed as an outpatient approximately two weeks later at which time the settings are re-titrated if necessary for optimal pain relief. After this time point adjustment by the care team is always possible but in practice rarely necessary. Patients are provided with a programmer device (presently an Apple iPod) which can communicate by radio with the IPG so that they can adjust the stimulus intensity for themselves from time to time if they so wish.

Once implanted, patients are under the care of the neuromodulation team indefinitely for any problems or issues related to their stimulator system.

\subsection{Device description}

The implanted system is manufactured by Abbott Laboratories under the trade name "Proclaim DRG".

The system components used are:

1) Proclaim DRG implantable pulse generator, manufacturer's part number 3664ANS

2) Axium SlimTip lead, manufacturer's part number MN20450-90A or MN20450-50A

3) Optional if needed: Axium extension lead, manufacturer's part number MN20550-50

\subsection{CMM Arm: Continued Medical Management}

Control patients will continue on their pre-existing pain treatment. We will not alter medication as part of the trial, but would not seek to prevent appropriate changes being made by other clinicians as necessary.

PENTAGONS: Diabetic Peripheral Neuropathy Treatment with Dorsal Root Ganglion Stimulation.

IRAS Project ID: 226499 REC Reference: 18/SC/0146

PENTAGONStrial_Protocol_V4.0_31Jul2019.docx31Jul2019

Clinical Research Protocol Template version 13.0

(C) Copyright: The University of Oxford and Oxford University Hospitals NHS Foundation Trust 2016

Page 23 of 46 
PENTAGONS: Diabetic Peripheral Neuropathy Treatment with Dorsal Root Ganglion Stimulation.

IRAS Project ID: 226499 REC Reference: 18/SC/0146

PENTAGONStrial_Protocol_V4.0_31Jul2019.docx31Jul2019

Clinical Research Protocol Template version 13.0

(c) Copyright: The University of Oxford and Oxford University Hospitals NHS Foundation Trust 2016

Page 24 of 46 


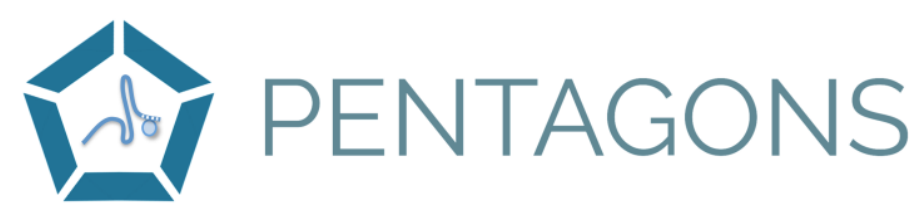

\section{SAFETY REPORTING}

The intervention arm in this trial will receive DRGS implantation. This procedure has become well established in the treatment of chronic neuropathic pain over the past 5 years. What is being investigated here is its use in patients with PDN.

\subsection{Definition of Adverse Device Effects (ADE's)}

Adverse device effects (an adverse event related to the use of an Investigational medical device) which may be linked to the device or trial procedures, but not deemed to be serious, will be reported.

The following list of ADE's, in relation to the DRGS procedure, will be reported:

- Dural puncture not resulting in extended hospital stay

- Wound pain without device infection

- Pocket site pain without device infection

- Pocket seroma without device infection

- Side effect/allergic reaction to procedure related medications or dressings

- Lead displacement or fracture where revision is not required.

\subsection{Reporting Procedures for Adverse Device Effects (ADE's)}

Trial centres will use a PENTAGONS paper ADE form to inform the PENTAGONS Trial Office of ADE's within twenty-four hours of becoming aware of them. This can be done via email sent to pentagons@nds.ox.ac.uk.

The seriousness and causality of ADE's (i.e. relationship to the trial device or procedure) will be assessed by the local investigator(s) on the ADE form. All ADE's, will be firstly reviewed by the PENTAGONS Trial Manager/office and discussed with the Chief Investigator, to determine whether the SAE is "related" and "unexpected"; as defined by the HRA guidance. These will then be assessed by the Nominated Clinician.

OCTRU safety reporting procedures will be followed at all times.

\subsection{Definition of Serious Adverse Events}

A serious adverse event is any untoward medical occurrence that:

- results in death

- is life-threatening

- requires inpatient hospitalisation or prolongation of existing hospitalisation

- results in persistent or significant disability/incapacity

- consists of a congenital anomaly or birth defect

PENTAGONS: Diabetic Peripheral Neuropathy Treatment with Dorsal Root Ganglion Stimulation.

IRAS Project ID: 226499 REC Reference: 18/SC/0146

PENTAGONStrial_Protocol_V4.0_31Jul2019.docx31Jul2019

Clinical Research Protocol Template version 13.0

CONFIDENTIAL

(C) Copyright: The University of Oxford and Oxford University Hospitals NHS Foundation Trust 2016

Page 25 of 46 


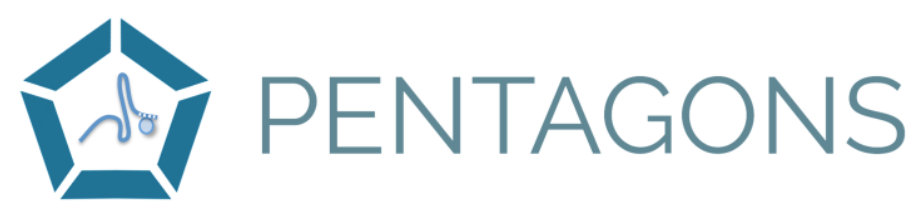

Other 'important medical events' may also be considered serious if they jeopardise the participant or require an intervention to prevent one of the above consequences.

NOTE: The term "life-threatening" in the definition of "serious" refers to an event in which the participant was at risk of death at the time of the event; it does not refer to an event which hypothetically might have caused death if it were more severe.

\subsection{Reporting Procedures for Serious Adverse Events}

Trial centres will use a PENTAGONS paper SAE form to inform the PENTAGONS Trial Office of SAEs within twenty-four hours of becoming aware of them (initial report). This can be done via email sent to pentagons@nds.ox.ac.uk.

The causality, expectedness and seriousness of SAEs (i.e. relationship to trial treatment) will be assessed by the local investigator(s) on the SAE form. All SAEs, will be reviewed by the PENTAGONS Trial Manager/office and discussed with the Chief Investigator, to determine whether the SAE is "related" and "unexpected" as defined by the HRA guidance. These will then be assessed by the Nominated Clinician.

The following will be reported as an SAE:

- Dural puncture resulting in extended hospital stay

- Wound pain with confirmed device infection

- Pocket site pain with confirmed device infection

- Pocket seroma with confirmed device infection

- Lead displacement or fracture where lead revision is required,

- Serious Adverse Device Effect (SADE - Adverse device effect that has resulted in any of the consequences characteristic of a serious adverse event)

The trial co-ordinating centre (Oxford) is responsible for reporting SAEs, where appropriate, to the Sponsor and ethics committee within required timelines. An SAE occurring to a participant will be reported to the REC that gave a favourable opinion of the study where in the opinion of the Chief Investigator the event was 'related' (resulted from administration of any of the research procedures) and 'unexpected' in relation to those procedures.

DRGS Implantation is known to have the following possible complications:

- Device infection

- Lead migration

- CSF leak

- Nerve damage due to direct injury as the lead is being inserted, or to bleeding around the nerves

\section{Postoperative device infections}

Will be specifically exempted from expedited reporting, so long as their frequency is not excessive. This is because infection is a recognised complication of stimulator implants owing to their construction from prosthetic material. Infection of such devices occurs at an average rate of

PENTAGONS: Diabetic Peripheral Neuropathy Treatment with Dorsal Root Ganglion Stimulation.

IRAS Project ID: 226499 REC Reference: 18/SC/0146

PENTAGONStrial_Protocol_V4.0_31Jul2019.docx31Jul2019

Clinical Research Protocol Template version 13.0

(C) Copyright: The University of Oxford and Oxford University Hospitals NHS Foundation Trust 2016

Page 26 of 46 


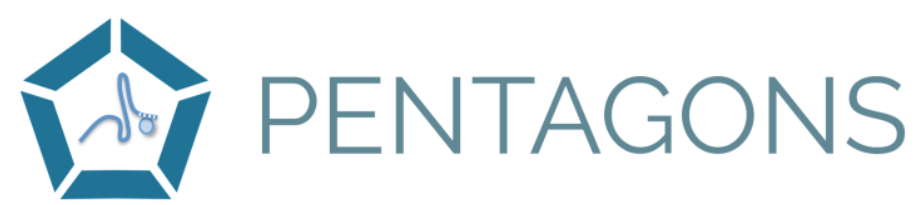

approximately $5 \%$ of cases overall, but diabetes is a predisposing factor for infection and one would expect a higher incidence in this setting. A large published series of 707 SCS cases (16) included 56 patients with diabetes, of whom five had a postoperative device infection, an incidence of $9 \%$. Using a binomial distribution, if the true incidence of infection is taken to be $9 \%$, then there is a $90 \%$ chance that in our sample of 28 implanted patients there should be four infections or fewer.

Therefore we will record and report device infections in routine fashion, but a report to the REC will be made if the number of infections exceeds four. The treatment for device infection is device removal.

\section{Lead migrations}

Will also be exempted from expedited reporting, so long as their frequency is not excessive, as they are also a recognised complication of neurostimulator implants. The best estimate of the frequency of lead migration comes from large series of percutaneous SCS lead implantations. In the series described above (16) the displacement rate was $22 \%$. In our sample of 28 patients, if the displacement rate is comparable then we would be $90 \%$ confident of seeing eight or fewer cases of lead displacement.

Therefore we will record and report lead displacements in routine fashion, but a report to the REC will be made if the number of lead migrations requiring lead revision exceeds eight. When migrations occur, it is standard practice to offer a lead replacement, and we will adhere to this standard practice during the study.

Details of post-operative device infections and lead migrations should however be reported on the appropriate form and monitored and managed according to standard clinical practice.

\section{N.B. Please note regarding lead complications:}

An SAE should ONLY be reported if the participant requires the revision of the lead; as only then will there be a need for hospitalisation. Otherwise this should be collected as an ADE.

Both Adverse Device Effects and Serious Adverse Events will be followed-up by the Investigator (clinic visits or telephone calls) until it has resolved or stabilised.

\subsection{Reporting Procedures for Unexpected Serious Adverse Events (SUSAR) and Unanticipated Serious Device Effects (USADE)}

Reports of 'related' and 'unexpected' SAEs (SUSAR - Suspected and Unexpected Serious Adverse Reaction - a serious adverse reaction, the nature and severity of which is not consistent with the information documented about the procedure or treatment in question) or an Unanticipated Serious Adverse Device Effect (USADE - Serious Adverse Device Effect which by its nature, incidence, severity or outcome has not

PENTAGONS: Diabetic Peripheral Neuropathy Treatment with Dorsal Root Ganglion Stimulation.

IRAS Project ID: 226499 REC Reference: 18/SC/0146

PENTAGONStrial_Protocol_V4.0_31Jul2019.docx31Jul2019

Clinical Research Protocol Template version 13.0

(C) Copyright: The University of Oxford and Oxford University Hospitals NHS Foundation Trust 2016

Page 27 of 46 


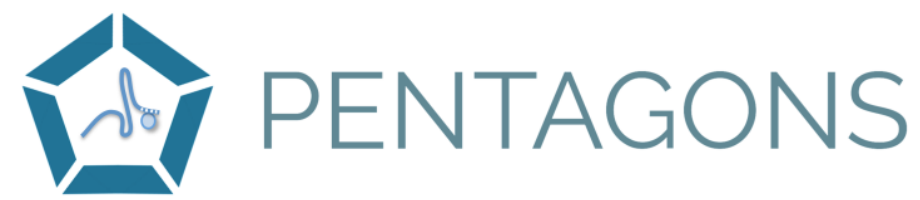

been identified in the current version of the risk analysis report) will be submitted within 15 working days of the Chief Investigator becoming aware of the event; using the HRA form found at https://www.hra.nhs.uk/documents/1087/safety-report-form-non-ctimp.docx.

OCTRU safety reporting procedures will be followed at all times.

\section{STATISTICS AND ANALYSIS}

Full details of the statistical analysis will be detailed in a separate statistical analysis plan (SAP) which will be drafted early in the trial and finalised prior to the primary analysis data lock. Stata (StataCorp LP) or other appropriate validated statistical software will be used for analysis. A summary of the planned statistical analysis is included here.

\subsection{The Number of Participants}

The primary outcome is treatment response, defined as a reduction of at least $50 \%$ on the VAS pain scale at 30 weeks post randomisation compared to baseline (a binary endpoint). We allow for the possibility that up to $10 \%$ of the control arm might achieve this (in the previous studies of spinal cord stimulation in diabetic neuropathy this was achieved in just $5 \%(8)$ and $7 \%(9)$ of the control arms). With $90 \%$ power, $5 \%$ 2-sided significance, and 1:1 randomisation, 25 patients per arm would be required to detect a $40 \%$ difference from $10 \%$ in the control arm to $50 \%$ in the implanted arm. A $50 \%$ responder rate is often taken as a marker of success in neuromodulation trials and based on available data it is a realistic goal: in the two previous trials of SCS in painful diabetic neuropathy the responder rates were $60 \%(8)$ and $59 \%(9)$, which corresponds respectively to a difference from controls of $55 \%$ and $52 \%$.

To allow for up to $10 \%$ loss to follow-up this figure is inflated to 28 patients per arm (56 in total).

\subsection{Description of Statistical Methods}

Participants will be included in the analysis on an intent-to-treat basis, that is, they will be analysed by the group to which they were randomized.

Baseline characteristics will be summarized overall and by treatment arm using descriptive statistics such as means and standard deviations (or medians and interquartile range if non-normally distributed) for continuous variables and counts and percentages for binary and categorical variables.

\subsection{Analysis of Outcome Measures}

The primary outcome is the proportion of participants achieving a response, defined as a $50 \%$ reduction in pain from baseline, at 30 weeks post randomisation. The two groups will be compared using the Chisquared test with supplementary analysis being undertaken using logistic regression adjusting for baseline pain, stratification factors and other important prognostic factors. The odds ratios together with $95 \%$ confidence intervals will be reported for both analyses. A 5\% two-sided significance level will be used. PENTAGONS: Diabetic Peripheral Neuropathy Treatment with Dorsal Root Ganglion Stimulation.

IRAS Project ID: 226499 REC Reference: 18/SC/0146

PENTAGONStrial_Protocol_V4.0_31Jul2019.docx31Jul2019

Clinical Research Protocol Template version 13.0

(C) Copyright: The University of Oxford and Oxford University Hospitals NHS Foundation Trust 2016

Page 28 of 46 


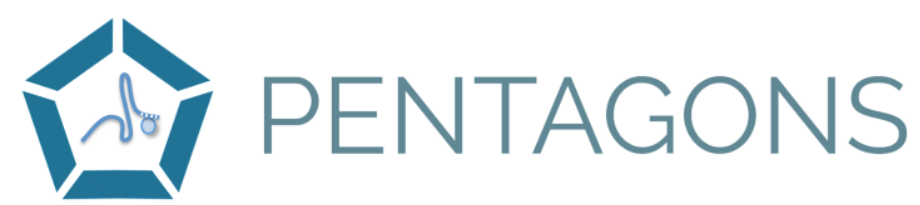

Other outcomes will be analysed using t-tests or ANCOVA adjusted for baseline values where appropriate for continuous variables with difference in means reported with $95 \%$ confidence intervals, and using chisquared tests or logistic regression for binary and categorical variables. Absolute and relative measures will be reported together with $95 \%$ confidence intervals.

If variables are not normally distributed, appropriate non-parametric tests will be undertaken.

\subsection{Physiology Sub study (see Appendix A)}

\section{DATA MANAGEMENT}

\subsection{Confidentiality}

All data will be handled in accordance with GDPR regulations.

Participants joining the trial will consent to give identifiable information that will include their name, email address, telephone contact numbers and home address in order to allow completion of the pain and QoL questionnaires and health resource use diaries. Participant's identifiable information will be kept secure on a University of Oxford network database, which is separate to the clinical database.

CRFs will not bear the participant's name, however their initials, year of birth and trial ID will be used for identification and this will be clearly explained to the participant in the PIS.

\subsection{Access to Data}

Direct access will be granted to authorised representatives from the Sponsor and host institution for monitoring and/or audit of the study to ensure compliance with regulations.

\subsection{Data collection tools}

Clinical data will be collected from sites on trial specific paper worksheets and then entered locally at sites onto an e-CRF using OpenClinica (www.openclinica.com) which is held in a Postgres database and can only be accessed by authorised users via the OpenClinica application. The OpenClinica application resides on a webserver hosted and managed by Oxford University's Medical Services Division IT Services department (http://www.imsu.ox.ac.uk/). The server is on the university's backbone network and is backed up nightly to a secure off-site location. Consent will be obtained from the participants to be able to share information for research and service improvement purposes, and prior to sharing, data will be anonymised.

Participants will be requested to complete pain and QoL questionnaires on-line using Lime Survey (a paper version will also be available). Participants will also be asked to complete three health resource use diaries by hand. Pre-paid envelopes will be provided, and the PENTAGONS Trial Office will coordinate the data entry of the resource use diaries.

PENTAGONS: Diabetic Peripheral Neuropathy Treatment with Dorsal Root Ganglion Stimulation.

IRAS Project ID: 226499 REC Reference: 18/SC/0146

PENTAGONStrial_Protocol_V4.0_31Jul2019.docx31Jul2019

Clinical Research Protocol Template version 13.0

(C) Copyright: The University of Oxford and Oxford University Hospitals NHS Foundation Trust 2016

Page 29 of 46 


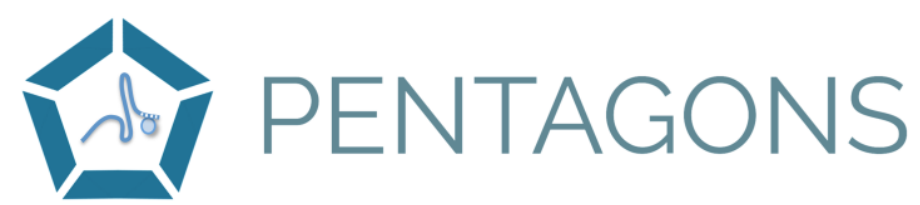

Text messaging and e-mail reminders to participants (who have consented to provide their telephone numbers and e-mail addresses) who have not completed/returned the pain and QoL questionnaires and resource utilisation diaries will be used to maximise completeness of data. The PENTAGONS Trial Office may also call participants if there are outstanding questionnaires or diaries not completed by the participant.

Pain scoring and QoL data will be collected using either standard pen or paper methods, whereby data will then be entered into a computer database (OpenClinica) centrally by the PENTAGONS trial office; or electronically via Lime Survey. Data from the resource diaries will be collected by pen or paper and then entered into OpenClinica centrally by the PENTAGONS Trial office.

Physiological data will be captured electronically by the software associated with each piece of test equipment, and stored on a University computer.

\subsection{Record Keeping}

Data will be stored under password protection on secure computers kept within the research facility. All data will be pseudonymised at the earliest opportunity and thereafter identified by code only. The code key will be stored separately in a secure location in an encrypted file.

Data will be backed up using University IT infrastructure and retained for at least 10 years. Metadata will be stored alongside the data detailing the methods used to generate the data, including the task used, the instrument used for testing and relevant setup information.

\section{QUALITY ASSURANCE PROCEDURES}

The trial will be conducted in accordance with the current approved protocol, Good Clinical Practice (GCP), relevant regulations and standard operating procedures.

Steps will be taken in the design, conduct and follow-up of the trial to reduce the occurrence of missing data. Steps have been taken to ensure that no unnecessary data are being collected, thus reducing participant burden. Staff are trained to collect data promptly and completely, and follow-up is encouraged after discontinuation of the randomized intervention and after individual assessments have been missed.

Regular monitoring will be performed according to GCP. Data will be evaluated for compliance with the protocol and accuracy in relation to source documents.

\section{ETHICAL AND REGULATORY CONSIDERATIONS}

\subsection{Declaration of Helsinki}

The Investigator will ensure that this study is conducted in accordance with the principles of the Declaration of Helsinki.

PENTAGONS: Diabetic Peripheral Neuropathy Treatment with Dorsal Root Ganglion Stimulation.

IRAS Project ID: 226499 REC Reference: 18/SC/0146

PENTAGONStrial_Protocol_V4.0_31Jul2019.docx31Jul2019

Clinical Research Protocol Template version 13.0

(C) Copyright: The University of Oxford and Oxford University Hospitals NHS Foundation Trust 2016

Page 30 of 46 


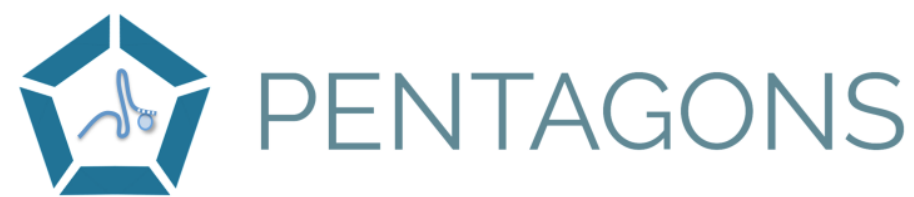

\subsection{Guidelines for Good Clinical Practice}

The Investigator will ensure that this study is conducted in accordance with relevant regulations and with Good Clinical Practice.

\subsection{Approvals}

The protocol, informed consent form, participant information sheet and any proposed advertising material will be submitted to an appropriate Research Ethics Committee (REC), and HRA for written approval.

The Investigator will submit and, where necessary, obtain approval from the above parties for all substantial amendments to the original approved documents.

\subsection{Reporting}

The $\mathrm{Cl}$ shall submit once a year throughout the study, or on request, an Annual Progress report to the REC Committee, HRA (where required) host organisation and Sponsor. In addition, an End of Study notification and final report will be submitted to the same parties.

\subsection{Participant Confidentiality}

The study staff will ensure that the participants' anonymity is maintained. The participants will be identified by a participant ID number on all study documents and any electronic database, with the exception of the CRF, where participant initials may be added. The participant will also be requested to consent to provide their e-mail address, mobile phone number and home address to enable electronic completion of pain and QoL questionnaires and paper completion of the health resource use diaries. If the participant does not wish to consent to electronic completion of the pain and QoL questionnaires, these can be completed on paper and will be posted by the PENTAGONS Trial Office. All documents will be stored securely and only accessible by study staff and authorised personnel.

The study will comply with the General Data Protection Regulation (GDPR) and Data Protection Act 2018, which requires data to be anonymised as soon as it is practical to do so.

\subsection{Expenses and Benefits}

Reasonable travel expenses for any visits additional to normal care will be reimbursed on production of receipts, or a mileage allowance provided as appropriate.

\subsection{Other Ethical Considerations}

No vulnerable participants or participants who are unable to consent for themselves will be included.

The risks associated with DRGS and with the neurophysiology assessments have been clearly outlined in the PIS, as well as the additional time taken to complete the questionnaires and undergo the assessments. Inconvenience to patients is minimised by the use of online data collection or postal questionnaires,

PENTAGONS: Diabetic Peripheral Neuropathy Treatment with Dorsal Root Ganglion Stimulation.

IRAS Project ID: 226499 REC Reference: 18/SC/0146

PENTAGONStrial_Protocol_V4.0_31Jul2019.docx31Jul2019

Clinical Research Protocol Template version 13.0

(C) Copyright: The University of Oxford and Oxford University Hospitals NHS Foundation Trust 2016 


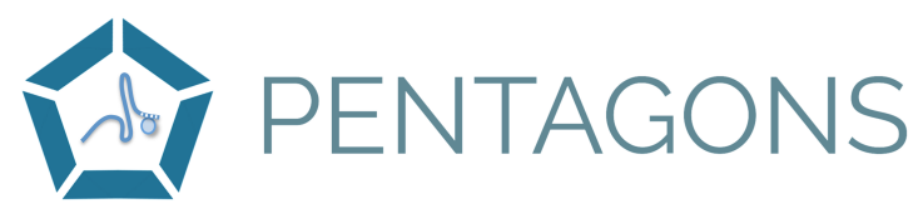

meaning that for the main study no additional visits are required over and above normal care. Additional visits are required for the physiology sub-study and this has been clearly stated in the PIS and will be reinforced verbally when discussing the sub-study with potential participants.

Assessment for possible DRGS includes MRI scanning of the lower back. In the unlikely event of seeing any structural abnormalities on an MRI scan, the scan will be checked by a clinical specialist. If the specialist feels that the abnormality was medically important, they will discuss the implications with the participant and arrange for further investigations as necessary. Participants will not be informed unless the doctor considers the finding has clear implications for their current or future health.

Inserting DRGS wires into the right place requires the use of X-ray fluoroscopy. lonising radiation can cause cell damage that may, after many years or decades, turn cancerous. The chances of this happening are the same whether DRGS is being done in this study or routinely outside it. X-rays can harm a developing baby so DRGS cannot be done in patients who are, or might be, pregnant.

Once the study is over, if the results are positive then participants in the control arm will be offered treatment. Patients for whom the device is ineffective will be offered device removal. Patients retaining a device will be followed up indefinitely by the neuromodulation team in their routine fashion. Further details regarding post-study care are given in Section 7.11.

\section{FINANCE AND INSURANCE}

\subsection{Funding}

The study is funded by Abbott Laboratories.

\subsection{Insurance}

The University has a specialist insurance policy in place which would operate in the event of any participant suffering harm as a result of their involvement in the research (Newline Underwriting Management Ltd, at Lloyd's of London). NHS indemnity operates in respect of the clinical treatment that is provided.

\section{PUBLICATION POLICY}

The Investigators will be involved in reviewing drafts of the manuscripts, abstracts, press releases and any other publications arising from the study. Authors will acknowledge that the study was funded by Abbott. Authorship will be determined in accordance with the ICMJE guidelines and other contributors will be acknowledged.

PENTAGONS: Diabetic Peripheral Neuropathy Treatment with Dorsal Root Ganglion Stimulation.

IRAS Project ID: 226499 REC Reference: 18/SC/0146

PENTAGONStrial_Protocol_V4.0_31Jul2019.docx31Jul2019

Clinical Research Protocol Template version 13.0

(C) Copyright: The University of Oxford and Oxford University Hospitals NHS Foundation Trust 2016

Page 32 of 46 


\section{Nof PENTAGONS}

\section{REFERENCES}

1. Zochodne DW. Diabetes mellitus and the peripheral nervous system: manifestations and mechanisms. Muscle \& nerve. 2007;36(2):144-66.

2. Davies M, Brophy S, Williams R, Taylor A. The prevalence, severity, and impact of painful diabetic peripheral neuropathy in type 2 diabetes. Diabetes care. 2006;29(7):1518-22.

3. Daousi C, MacFarlane IA, Woodward A, Nurmikko TJ, Bundred PE, Benbow SJ. Chronic painful peripheral neuropathy in an urban community: a controlled comparison of people with and without diabetes. Diabetic medicine : a journal of the British Diabetic Association. 2004;21(9):976-82.

4. Ziegler D, Gries FA, Spuler M, Lessmann F. The epidemiology of diabetic neuropathy. Diabetic Cardiovascular Autonomic Neuropathy Multicenter Study Group. Journal of diabetes and its complications. 1992;6(1):49-57.

5. Snedecor SJ, Sudharshan L, Cappelleri JC, Sadosky A, Mehta S, Botteman M. Systematic review and meta-analysis of pharmacological therapies for painful diabetic peripheral neuropathy. Pain practice : the official journal of World Institute of Pain. 2014;14(2):167-84.

6. Jensen TS, Backonja MM, Hernandez Jimenez S, Tesfaye S, Valensi P, Ziegler D. New perspectives on the management of diabetic peripheral neuropathic pain. Diabetes \& vascular disease research. 2006;3(2):108-19.

7. Dworkin RH, O'Connor AB, Audette J, Baron R, Gourlay GK, Haanpaa ML, et al. Recommendations for the pharmacological management of neuropathic pain: an overview and literature update. Mayo Clinic proceedings. 2010;85(3 Suppl):S3-14.

8. de Vos CC, Meier K, Zaalberg PB, Nijhuis HJ, Duyvendak W, Vesper J, et al. Spinal cord stimulation in patients with painful diabetic neuropathy: a multicentre randomized clinical trial. Pain. 2014;155(11):2426-31.

9. Slangen R, Schaper NC, Faber CG, Joosten EA, Dirksen CD, van Dongen RT, et al. Spinal cord stimulation and pain relief in painful diabetic peripheral neuropathy: a prospective two-center randomized controlled trial. Diabetes care. 2014;37(11):3016-24.

10. Deer TR, Levy RM, Kramer J, Poree L, Amirdelfan K, Grigsby E, et al. Dorsal root ganglion stimulation yielded higher treatment success rate for complex regional pain syndrome and causalgia at 3 and 12 months: a randomized comparative trial. Pain. 2016.

11. Wu M, Linderoth B, Foreman RD. Putative mechanisms behind effects of spinal cord stimulation on vascular diseases: a review of experimental studies. Autonomic neuroscience : basic \& clinical. 2008;138(1-2):9-23.

12. Wukich DK, Crim BE, Frykberg RG, Rosario BL. Neuropathy and poorly controlled diabetes increase the rate of surgical site infection after foot and ankle surgery. J Bone Joint Surg Am. 2014;96(10):832-9.

13. Han HS, Kang SB. Relations between long-term glycemic control and postoperative wound and infectious complications after total knee arthroplasty in type 2 diabetics. Clin Orthop Surg. 2013;5(2):118-23.

14. Perkins BA, Olaleye D, Zinman B, Bril V. Simple screening tests for peripheral neuropathy in the diabetes clinic. Diabetes care. 2001;24(2):250-6.

15. Bril V, Perkins BA. Validation of the Toronto Clinical Scoring System for diabetic polyneuropathy. Diabetes care. 2002;25(11):2048-52.

16. Mekhail NA, Mathews M, Nageeb F, Guirguis M, Mekhail MN, Cheng J. Retrospective review of 707 cases of spinal cord stimulation: indications and complications. Pain practice : the official journal of World Institute of Pain. 2011;11(2):148-53.

PENTAGONS: Diabetic Peripheral Neuropathy Treatment with Dorsal Root Ganglion Stimulation.

IRAS Project ID: 226499 REC Reference: 18/SC/0146

PENTAGONStrial_Protocol_V4.0_31Jul2019.docx31Jul2019

Clinical Research Protocol Template version 13.0

(C) Copyright: The University of Oxford and Oxford University Hospitals NHS Foundation Trust 2016

Page 33 of 46 
17. APPENDIX A: SUB-STUDY

\section{Background}

The PENTAGONS trial is designed to assess the effectiveness of Dorsal Root Ganglion Stimulation (DRGS) in reducing pain in the feet that results from diabetic peripheral neuropathy. Another common and serious complication of diabetic peripheral neuropathy is foot ulceration. A number of factors contribute to the genesis of ulcers including impaired sensation, reduced perfusion, and poor wound healing. Not uncommonly, persistent ulceration presages amputation. The treatment of diabetic ulcers and amputations costs the NHS approximately $\mathrm{f650}$ million per year (https://www.diabetes.org.uk/ Documents/nhs-diabetes/footcare/footcare-for-people-with diabetes.pdf).

Spinal Cord Stimualtion (SCS) has been observed to cause peripheral vasodilatation (11). This is thought to be the result of the stimulation causing retrograde nerve impulses in sensory nerve fibres; these cause the nerve endings in the skin to release substances that dilate small blood vessels. This raises the possibility that DRGS may also improve perfusion, and in theory the stimulation site in DRGS should produce a superior effect to SCS. Evaluating the possible beneficial effects of DRGS on skin perfusion will be an important secondary aim of this study.

Diabetic neuropathy occurs because the diabetes damages small blood vessels that supply nerves. As well as pain, it causes impairment of sensation and loss of autonomic function. It is possible that if DRGS improves skin perfusion, it might also improve nerve perfusion and function. We will therefore also quantify these deficits and see what effect, if any, DRGS has on them.

\section{Study Proposal}

This nested sub-study will include a randomized subset of 20-24 patients (10-12 DRGS and 10-12 control) at the John Radcliffe Hospital, Oxford. A member of the clinical or research team will approach participants enrolled in the main PENTAGONS trial. Additional verbal and written information will be provided regarding the physiology sub study, after and only if the patient has agreed to take part in the main trial, and patients will have the option of participating in the main trial, with or without participation in the sub study. Consent to the sub study will be recorded on a separate form.

As well as the baseline questionnaires of the main study, those participating in the Physiology sub-study will have baseline measurements of:

- Cutaneous tissue oxygenation (tcPO2) and perfusion (laser Doppler). tcPO2 involves placing a sensor on the skin surface which non-invasively measures oxygen levels in the skin underneath it, while laser Doppler involves shining a laser light at the skin and examining the light reflected back to a sensor. The laser is low power and not damaging.

- Quantitative Sensory Testing (QST) to measure impairment in both large fibre (vibration) and small fibre (temperature) modalities. These tests are done by placing a warming, cooling, or vibrating pad against the skin; temperature/vibration amplitude are gradually changed and we measure the participant's ability to detect the change.

PENTAGONS: Diabetic Peripheral Neuropathy Treatment with Dorsal Root Ganglion Stimulation.

IRAS Project ID: 226499 REC Reference: 18/SC/0146

PENTAGONStrial_Protocol_V4.0_31Jul2019.docx31Jul2019

Clinical Research Protocol Template version 13.0

(C) Copyright: The University of Oxford and Oxford University Hospitals NHS Foundation Trust 2016

Page 34 of 46 


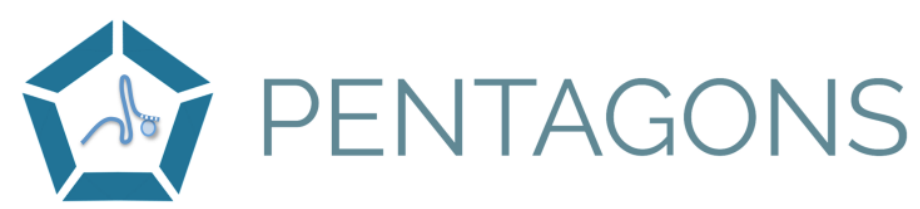

- Tests of sympathetic nerve activity (Galvanic skin response). Galvanic skin response measures the dryness of the skin by measuring electrical conductivity over its surface.

The follow up time points for the Physiology sub-study will be identical to those for the main study $(8,18$, and 30 weeks). At each follow up time point the same physiological testing that was done at baseline will be repeated. This will require attendance in person for testing. To minimise inconvenience, the first follow up testing in the surgical arm will be done at the time of the 2 week postoperative review. Apart from the physiological testing the follow up of patients in this sub-study will be identical to that of other patients in the main study. To ensure consistency with participants not in the sub-study, those who are in the substudy will still complete their questionnaires online or by post.

\section{Eligibility}

Participants in the main PENTAGONS study at the lead site will be eligible for inclusion in the PENTAGONS physiology sub study.

\section{Sample size}

There is no data available to underpin an accurate sample size calculation. The planned recruitment to the sub study of 10-12 patients from each arm of the main study reflects the estimated numbers needed to detect changes in the physiological measurements, and the anticipated recruitment to the main study at the lead centre. Specialist equipment will be used to make precise, objective measurements of linear physiological variables, and fewer patients will be needed than for the primary outcome measure of the main study.

\section{Outcome measures}

Changes in nerve function (sensory, autonomic) and cutaneous perfusion and oxygenation in the lower limb.

\section{Risks}

Sub study procedures are non-invasive and pose no extra risk to the patient.

\section{Statistics}

Analysis of the data from the sub study will be performed by members of the study team with relevant physiological expertise. Data will be described and compared using appropriate parametric (if normally distributed) or nonparametric statistics, both cross sectionally (DRGS versus control) and longitudinally (change over time).

PENTAGONS: Diabetic Peripheral Neuropathy Treatment with Dorsal Root Ganglion Stimulation.

IRAS Project ID: 226499 REC Reference: 18/SC/0146

PENTAGONStrial_Protocol_V4.0_31Jul2019.docx31Jul2019

Clinical Research Protocol Template version 13.0

(C) Copyright: The University of Oxford and Oxford University Hospitals NHS Foundation Trust 2016

Page 35 of 46 
PENTAGONS: Diabetic Peripheral Neuropathy Treatment with Dorsal Root Ganglion Stimulation.

IRAS Project ID: 226499 REC Reference: 18/SC/0146

PENTAGONStrial_Protocol_V4.0_31Jul2019.docx31Jul2019

Clinical Research Protocol Template version 13.0

(c) Copyright: The University of Oxford and Oxford University Hospitals NHS Foundation Trust 2016

Page 36 of 46 


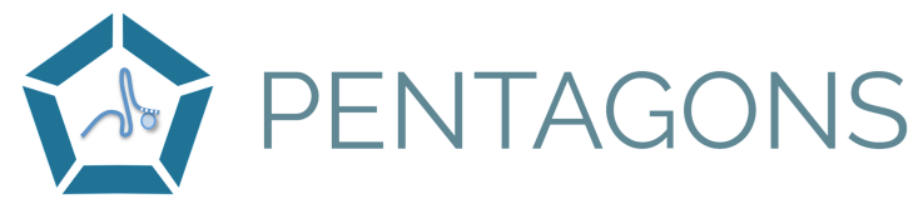

\section{APPENDIX B: STUDY FLOW CHART}

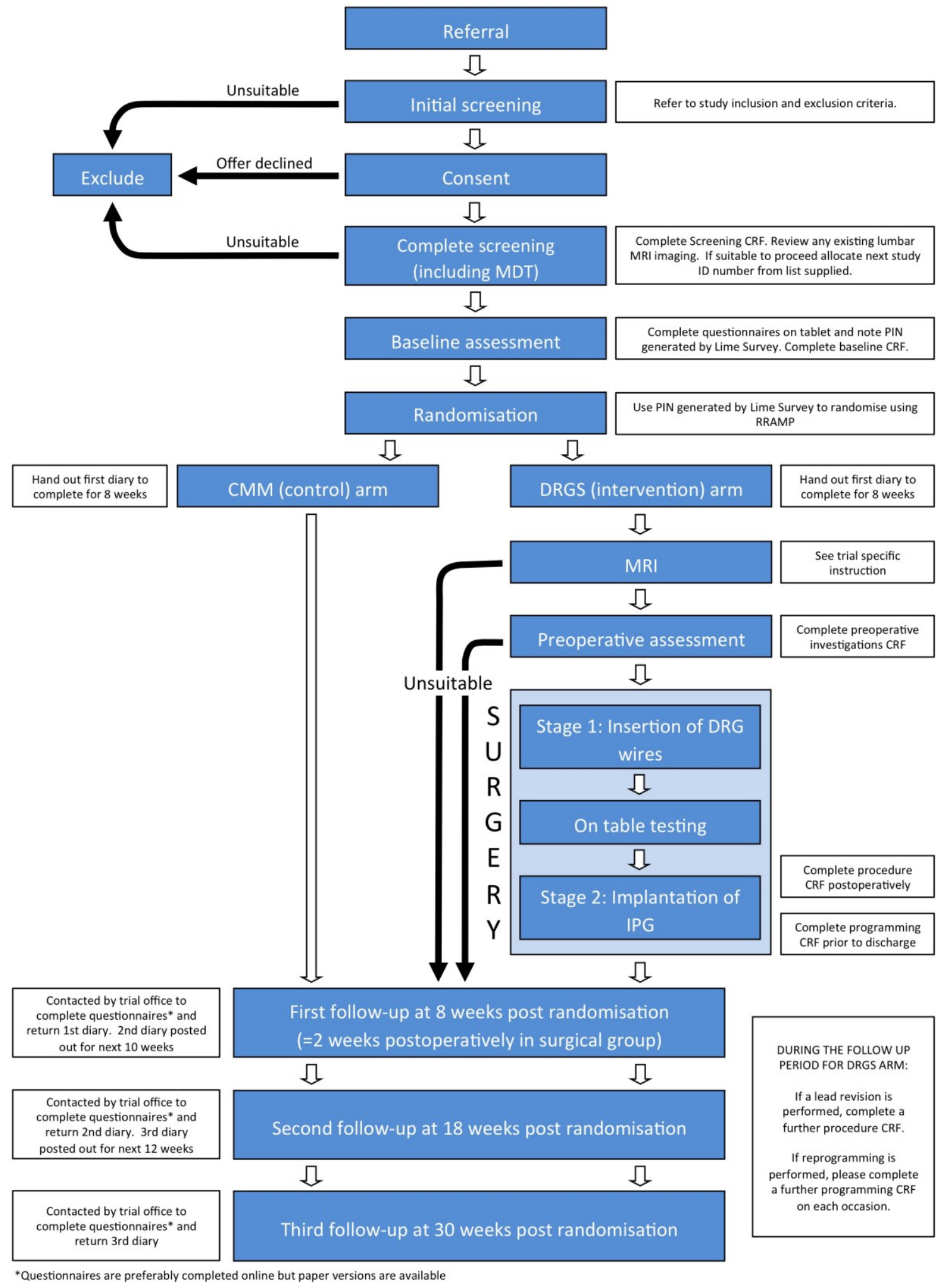

V11

PENTAGONS: Diabetic Peripheral Neuropathy Treatment with Dorsal Root Ganglion Stimulation.

IRAS Project ID: 226499 REC Reference: 18/SC/0146

PENTAGONStrial_Protocol_V4.0_31Jul2019.docx31Jul2019

Clinical Research Protocol Template version 13.0

CONFIDENTIAL

(c) Copyright: The University of Oxford and Oxford University Hospitals NHS Foundation Trust 2016 


\section{Nof PENTAGONS}

19. APPENDIX C: SCHEDULE OF STUDY PROCEDURES

\begin{tabular}{|c|c|c|c|c|c|c|}
\hline \multirow[b]{3}{*}{ Procedures } & \multicolumn{6}{|c|}{ Visits } \\
\hline & $\begin{array}{l}\text { Screening } \\
\text { \& Consent }\end{array}$ & Baseline & DRGS & $\begin{array}{c}\text { Follow up } \\
\text { (Visit 1) }\end{array}$ & $\begin{array}{c}\text { Follow up } \\
\text { (Visit 2) }\end{array}$ & $\begin{array}{c}\text { Follow up } \\
\text { (Visit 3) }\end{array}$ \\
\hline & $\begin{array}{l}\text { Within } 12 \\
\text { weeks prior } \\
\text { to baseline } \\
\text { assessment }\end{array}$ & $\begin{array}{c}\text { \& } \\
\text { Randomise }\end{array}$ & $\begin{array}{l}\sim 6 \text { weeks } \\
\text { after RND }\end{array}$ & $\begin{array}{l}8 \text { weeks } \\
\text { from RND }\end{array}$ & $\begin{array}{l}18 \text { weeks } \\
\text { from RND }\end{array}$ & $\begin{array}{l}30 \text { weeks } \\
\text { from RND }\end{array}$ \\
\hline Screening & $X$ & & & & & \\
\hline $\begin{array}{l}\text { Enrolment and } \\
\text { informed } \\
\text { consent }\end{array}$ & $X$ & & & & & \\
\hline $\begin{array}{c}\text { Randomisation } \\
\text { (RND) }\end{array}$ & & $x^{1}$ & & & & \\
\hline $\begin{array}{l}\text { MRI/ECG/Pre- } \\
\text { procedure } \\
\text { checks }\end{array}$ & & $x^{3}$ & & & & \\
\hline $\begin{array}{c}\text { Stimulator } \\
\text { implantation } \\
\text { and initial } \\
\text { titration }\end{array}$ & & & $X$ & & & \\
\hline $\begin{array}{c}\text { Postoperative } \\
\text { check }\end{array}$ & & & & $X$ & & \\
\hline Pain scores & & $x^{2}$ & & $x^{5}$ & $x^{5}$ & $x^{5}$ \\
\hline $\begin{array}{l}\text { Quality of life } \\
\text { measures }\end{array}$ & & $x$ & & $* x^{5}$ & $x^{5}$ & $x^{5}$ \\
\hline $\begin{array}{l}\text { Medication } \\
\text { levels }\end{array}$ & & $X$ & & $x^{7}$ & $x^{7}$ & $x^{7}$ \\
\hline $\begin{array}{c}\text { Resource } \\
\text { utilisation } \\
\text { diaries issued }\end{array}$ & & $X$ & & $X$ & $X$ & \\
\hline $\begin{array}{l}\text { Collection of } \\
\text { resource } \\
\text { utilisation } \\
\text { diaries }\end{array}$ & & & & $x^{6}$ & $x^{6}$ & $x^{6}$ \\
\hline $\begin{array}{l}\text { Physiological } \\
\text { testing (Sub- } \\
\text { study) }\end{array}$ & & $x^{4}$ & & $x^{4}$ & $x^{4}$ & $x^{4}$ \\
\hline
\end{tabular}

PENTAGONS: Diabetic Peripheral Neuropathy Treatment with Dorsal Root Ganglion Stimulation.

IRAS Project ID: 226499 REC Reference: 18/SC/0146

PENTAGONStrial_Protocol_V4.0_31Jul2019.docx31Jul2019

Clinical Research Protocol Template version 13.0

(C) Copyright: The University of Oxford and Oxford University Hospitals NHS Foundation Trust 2016 


\section{Af PENTAGONS}

1 Randomisation (RND) will occur after the baseline scoring/testing in this column has been completed.

2 Collect PCS score at BASELINE ONLY

3 For those randomised to the DRGS arm only

4 This will be performed in a subset of participants at the Lead site ONLY

5 This data will be collected online or postally

*5 only the EQ-5D-5L Quality of life measure is collected at this time point, and this is used solely for the healthcare economic analysis.

6 PENTAGONS trial office will contact participants to return the diaries using the prepaid envelopes provided. 7 Medication levels will be collected via the resource use diaries; apart from BASELINE where they will also be collected on the CRF

PENTAGONS: Diabetic Peripheral Neuropathy Treatment with Dorsal Root Ganglion Stimulation.

IRAS Project ID: 226499 REC Reference: 18/SC/0146

PENTAGONStrial_Protocol_V4.0_31Jul2019.docx31Jul2019

Clinical Research Protocol Template version 13.0

(C) Copyright: The University of Oxford and Oxford University Hospitals NHS Foundation Trust 2016

Page 39 of 46 


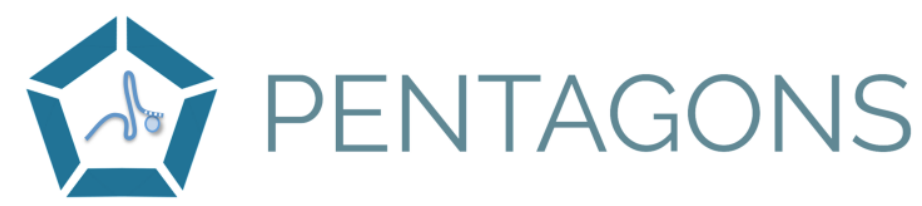

20. APPENDIX D: MICHIGAN NEUROPATHY SCREENING INSTRUMENT (MNSI)

\section{Patient Version \\ MICHIGAN NEUROPATHY SCREENING INSTRUMENT}

A. History (To be completed by the person with diabetes)

Please take a few minutes to answer the following questions about the feeling in your legs and feet. Check yes or no based on how you usually feel. Thank you.

1. Are you legs and/or feet numb?

2. Do you ever have any burning pain in your legs and/or feet?

$\square$ Yes $\square$ No

3. Are your feet too sensitive to touch?

$\square$ Yes $\square$ No

4. Do you get muscle cramps in your legs and/or feet?

$\square$ Yes $\square$ No

5. Do you ever have any prickling feelings in your legs or feet?

$\square$ Yes $\square$ No

6. Does it hurt when the bed covers touch your skin?

Yes $\square$ No

7. When you get into the tub or shower, are you able to tell the hot water from the cold water?

Yes $\square$ No

8. Have you ever had an open sore on your foot?

$\square$ Yes $\square$ No

9. Has your doctor ever told you that you have diabetic neuropathy?

$\square$ Yes $\square$ No

10. Do you feel weak all over most of the time?

$\square$ Yes $\square$ No

11. Are your symptoms worse at night?

$\square$ Yes $\square$ No

12. Do your legs hurt when you walk?

$\square$ Yes $\square$ No

13. Are you able to sense your feet when you walk?

$\square$ Yes $\square$ No

14. Is the skin on your feet so dry that it cracks open?

$\square$ Yes $\square$ No

15. Have you ever had an amputation?

$\square$ Yes $\square$ No

$\square$ Yes $\square$ No

Total: 


\section{位 PENTAGONS}

\section{MICHIGAN NEUROPATHY SCREENING INSTRUMENT}

B. Physical Assessment (To be completed by health professional)

1. Appearance of Feet

Right

a. Normal $\quad \square 0$ Yes $\quad \square_{1}$ No

b. If no, check all that apply.

Deformities

Dry skin, callus

Infection

Fissure

Other

specify:

Right

2. Ulceration

Absent Present

\section{Left}

Normal $\square$ o Yes $\quad \square$ i No

If no, check all that apply:

Deformities $\square$

Dry skin, callus

Infection

Fissure

Other

specify:

\begin{tabular}{ll}
\hline & Left \\
Absent & \\
$\square$ & Present
\end{tabular}

3. Ankle Reflexes

Present/

Present Reinforcement Absent

Present/

Present Reinforcement Absent
$\square 0.5$
$\square 1$

$\square 0$

$\square 0.5$

$\square 1$

\begin{tabular}{|c|c|c|}
\hline & & \\
\hline $\begin{array}{l}\text { Vibration } \\
\text { perception at } \\
\text { great toe }\end{array}$ & $\square 0$ & $\square 0.5$ \\
\hline
\end{tabular}

5. Monofilament Normal Reduced Absent

$\square 0 \quad \square 0.5 \quad \square 1$

Signature:

Total Score $/ 10$ Points

MNSI, (c) University of Michigan, 2000

PENTAGONS: Diabetic Peripheral Neuropathy Treatment with Dorsal Root Ganglion Stimulation.

IRAS Project ID: 226499 REC Reference: 18/SC/0146

PENTAGONStrial_Protocol_V4.0_31Jul2019.docx31Jul2019

Clinical Research Protocol Template version 13.0

(C) Copyright: The University of Oxford and Oxford University Hospitals NHS Foundation Trust 2016 


\section{位 PENTAGONS}

\section{APPENDIX E: FUNDING}

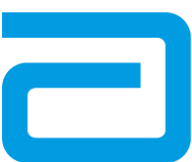

Abbott

5050 Nathan Lane N

Plymouth, MN 55442

T: 6517563571

SPRB_Review@sjm.com

\section{Abbott}

August 2, 2017

James Fitzgerald, MD, PhD

Nuffield Department of Surgical Sciences, University of Oxford

Department of Neurosurgery, Level 3, West Wing

John Radcliffe Hospital

Headley Way, Headington

Oxford

United Kingdom

Re: Study ID\#17167 PENTAGONS: Diabetic Peripheral Neuropathy Treatment with Dorsal Root Ganglion Stimulation -- a Randomised Controlled Trial.

Dear Dr. Fitzgerald,

Our Study Proposal Review Board (SPRB) recently reviewed your study proposal entitled "PENTAGONS". I am happy to inform you that the proposal was approved for $£ 295,000$.

William Cusack (wcusack@sjm.com) will be your point of contact within Abbott for this study and will follow-up soon with next steps, which will include the development of a research agreement outlining the terms of our support.

Please note that our support will not be in place indefinitely. This is contingent on Abbott and the research site and/or principal investigator entering into an agreement. We expect to be able to come to agreement on the terms of our support within 3 months, and the study to thereafter progress reasonably as will be outlined within the agreement.

Thank you for reaching out to Abbott with your study idea. Please do not hesitate to contact me if you have any questions or concerns as we look forward to the initiation of this very interesting and important research.

Sincerely,

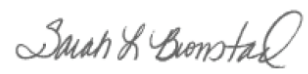

Sarah Bronstad

Project Manager, Investigator Initiated Studies

SPRB Chairman

Cc: William Cusack

PENTAGONS: Diabetic Peripheral Neuropathy Treatment with Dorsal Root Ganglion Stimulation. 


\section{서 PENTAGONS}

22. APPENDIX F: AMENDMENT HISTORY

\begin{tabular}{|c|c|c|c|c|}
\hline $\begin{array}{l}\text { Amendment } \\
\text { No. }\end{array}$ & $\begin{array}{l}\text { Protocol } \\
\text { Version } \\
\text { No. }\end{array}$ & $\begin{array}{l}\text { Date } \\
\text { issued }\end{array}$ & Author(s) of changes & Details of Changes made \\
\hline 01 & 3.0 & 13 Nov2018 & $\begin{array}{l}\text { James Fitzgerald, Ines } \\
\text { Rombach, Jane } \\
\text { Wolstenholme, Damian } \\
\text { Haywood and Jo Cook }\end{array}$ & $\begin{array}{l}\text { 1. Clarification of } \\
\text { Secondary outcomes } \\
\text { 2.Clarification of time } \\
\text { points of questionnaires } \\
\text { and resource utilisation } \\
\text { diaries in relation to both } \\
\text { trial arms } \\
\text { 3. Addition of another } \\
\text { time point for the health } \\
\text { resource utilisation diary } \\
\text { 4. Toronto Clinical } \\
\text { Scoring System, used at } \\
\text { Screening, changed to } \\
\text { the Michigan } \\
\text { Neuropathy Screening } \\
\text { Instrument and added to } \\
\text { the appendices. } \\
\text { 5. Addition and } \\
\text { amendment to both the } \\
\text { inclusion and exclusion } \\
\text { criteria } \\
\text { 6. Amendment to the } \\
\text { review of the MRI prior } \\
\text { to the DRGS procedure } \\
\text { and the creation of a } \\
\text { Trial Specific Instruction } \\
\text { 7. McGill Pain } \\
\text { Questionnaire removed } \\
\text { from PROMS and } \\
\text { changed to Brief Pain } \\
\text { Inventory. } \\
\text { 8. Addition of the } \\
\text { Medical Outcomes Sleep } \\
\text { Survey to the PROMS } \\
\text { 9. Addition of Lime } \\
\text { survey for the electronic } \\
\text { collection of the data for } \\
\text { the questionnaires } \\
\text { 10. Addition of the } \\
\text { Salford Royal NHS } \\
\text { Foundation Trust in } \\
\text { Manchester as a site. }\end{array}$ \\
\hline
\end{tabular}

PENTAGONS: Diabetic Peripheral Neuropathy Treatment with Dorsal Root Ganglion Stimulation.

IRAS Project ID: 226499 REC Reference: 18/SC/0146

PENTAGONStrial_Protocol_V4.0_31Jul2019.docx31Jul2019

Clinical Research Protocol Template version 13.0

CONFIDENTIAL

(C) Copyright: The University of Oxford and Oxford University Hospitals NHS Foundation Trust 2016 


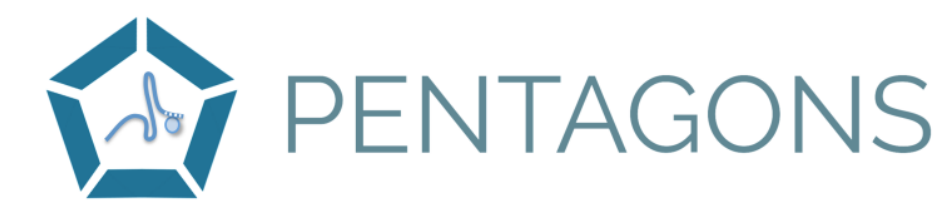

\begin{tabular}{|c|c|c|c|c|}
\hline & & & & $\begin{array}{l}\text { 11. Addition of PIC sites } \\
\text { (GP surgeries in the } \\
\text { Thames Valley and South } \\
\text { Midlands CRN) for the } \\
\text { LEAD site only } \\
\text { 12. Clarification and } \\
\text { Amendment of the } \\
\text { 'Safety Reporting } \\
\text { Section' } \\
\text { 13. Clarification of the } \\
\text { 'Discontinuation/ } \\
\text { Withdrawal' section } \\
\text { 14. Clarification of the } \\
\text { Data Management } \\
\text { section with the } \\
\text { introduction of GDPR } \\
\text { 15. Addition of the Sub- } \\
\text { study as an appendix16. } \\
\text { Amendment and } \\
\text { clarification to the study } \\
\text { flow chart. } \\
\text { 17. Amendment and } \\
\text { Clarification of the } \\
\text { Schedule of study } \\
\text { procedures }\end{array}$ \\
\hline $\begin{array}{l}\text { Amendment } \\
\text { No. }\end{array}$ & $\begin{array}{l}\text { Protocol } \\
\text { Version } \\
\text { No. }\end{array}$ & $\begin{array}{l}\text { Date } \\
\text { issued }\end{array}$ & Author(s) of changes & Details of Changes made \\
\hline 02 & $\begin{array}{l}\text { Currently } \\
\text { Version } 3.2 \\
\text { to send to } \\
\text { the } \\
\text { Sponsor) } \\
\text { when } \\
\text { approved } \\
\text { by Sponsor } \\
\text { will } \\
\text { become } \\
\text { V4.0 }\end{array}$ & $\begin{array}{l}\text { To be } \\
\text { added } \\
\text { when V4.0 } \\
\text { approved } \\
\text { by Sponsor } \\
\text { (currently } \\
\text { V3.2 }\end{array}$ & $\begin{array}{l}\text { James Fitzgerald, Jane } \\
\text { Wolstenholme, Cathy Qi, } \\
\text { Ines Rombach, Damian } \\
\text { Haywood and Jo Cook }\end{array}$ & $\begin{array}{l}\text { 1. Addition of St } \\
\text { George's University } \\
\text { Hospital, London } \\
\text { as a participating site } \\
\text { 2. Addition of GP PICs for } \\
\text { all participating sites in } \\
\text { order to maximise } \\
\text { recruitment (addition of } \\
\text { their associated CRN's). } \\
\text { 3. Clarification of } \\
\text { processes and additional } \\
\text { routes for identifying } \\
\text { participants for } \\
\text { recruitment to Section } \\
7.1 \text { 'Recruitment' }\end{array}$ \\
\hline
\end{tabular}

PENTAGONS: Diabetic Peripheral Neuropathy Treatment with Dorsal Root Ganglion Stimulation.

IRAS Project ID: 226499 REC Reference: 18/SC/0146

PENTAGONStrial_Protocol_V4.0_31Jul2019.docx31Jul2019

Clinical Research Protocol Template version 13.0

CONFIDENTIAL

(C) Copyright: The University of Oxford and Oxford University Hospitals NHS Foundation Trust 2016 


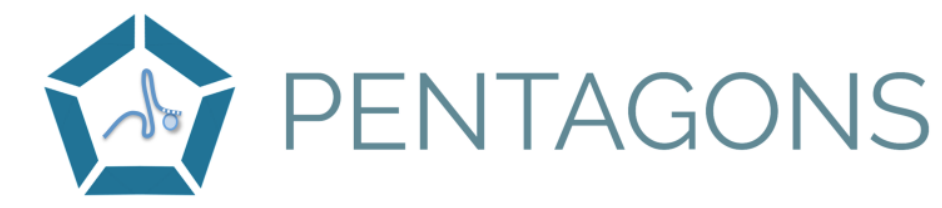

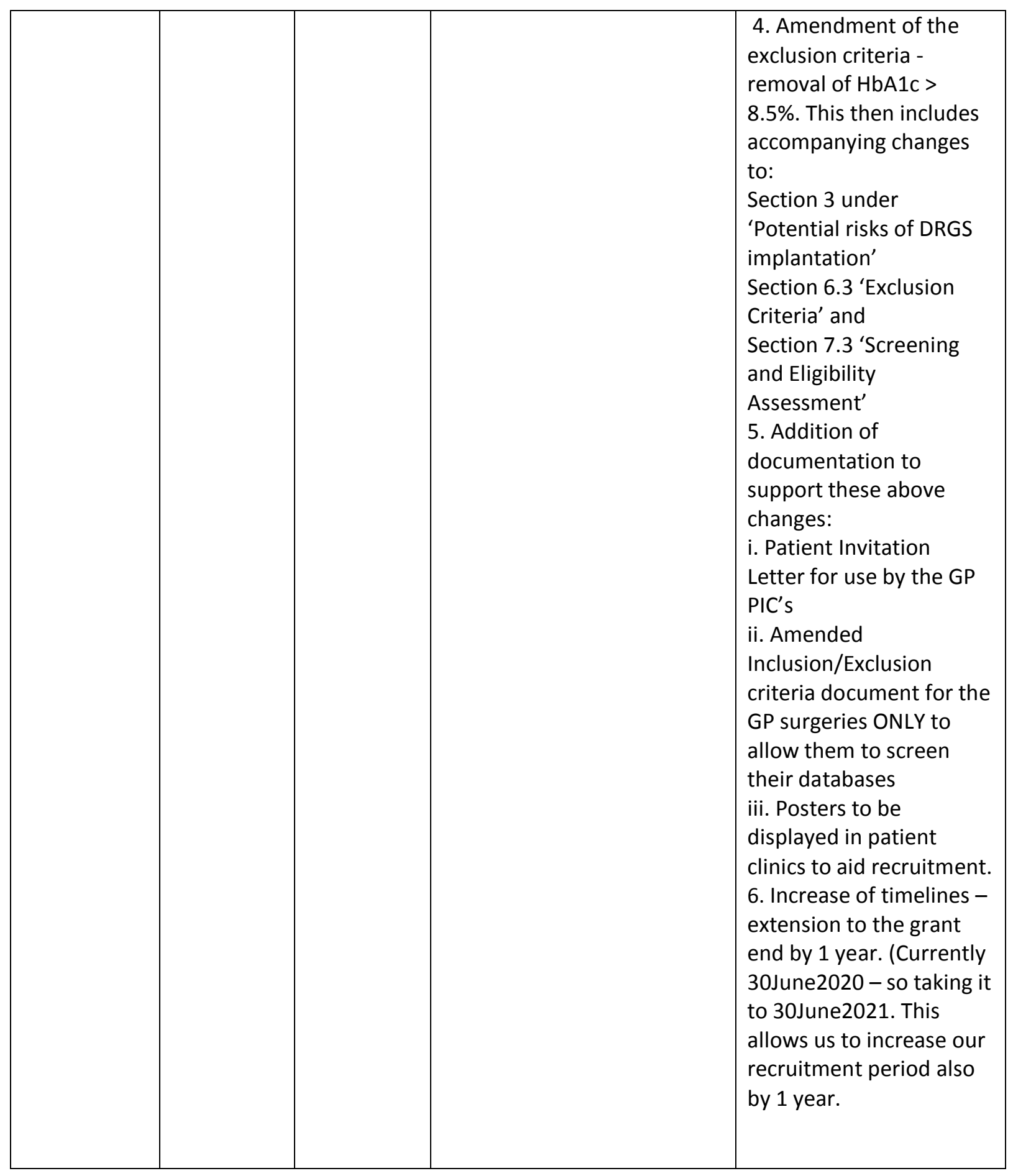

PENTAGONS: Diabetic Peripheral Neuropathy Treatment with Dorsal Root Ganglion Stimulation.

IRAS Project ID: 226499 REC Reference: 18/SC/0146

PENTAGONStrial_Protocol_V4.0_31Jul2019.docx31Jul2019

Clinical Research Protocol Template version 13.0

(C) Copyright: The University of Oxford and Oxford University Hospitals NHS Foundation Trust 2016 
PENTAGONS: Diabetic Peripheral Neuropathy Treatment with Dorsal Root Ganglion Stimulation.

IRAS Project ID: 226499 REC Reference: 18/SC/0146

PENTAGONStrial_Protocol_V4.0_31Jul2019.docx31Jul2019

Clinical Research Protocol Template version 13.0

(c) Copyright: The University of Oxford and Oxford University Hospitals NHS Foundation Trust 2016

Page 46 of 46 